\title{
Une charpente sculptée du Xle siècle : l'église Saint-Pierre de Neufmarché-en-Lyons (Seine-Maritime)
}

In: Bulletin Monumental. Tome 161 №2, année 2003. pp. 101-115.

\section{Citer ce document / Cite this document :}

Epaud Frédéric, Bernard Vincent. Une charpente sculptée du Xle siècle : l'église Saint-Pierre de Neufmarché-en-Lyons (SeineMaritime). In: Bulletin Monumental. Tome $161 \mathrm{~N}^{\circ} 2$, année 2003. pp. 101-115.

doi : 10.3406/bulmo.2003.1183

http://www.persee.fr/web/revues/home/prescript/article/bulmo_0007-473X_2003_num_161_2_1183 


\section{Abstract}

An eleventh-century roofframework with a sculpted croup : the church of Saint-Pierre de Neufmarchéen-Lyons, by Frédéric Épaud and Vincent Bernard.

In the mid eleventh century, the church of Saint-Pierre de Neufmarché-en-Lyons (Seine-Maritime) was the collegiate church of the Grandmesnil family who were governors of the place de Neufmarché. Converted into a priory as a dependency of the abbey of Saint Evroult en Ouche around 1065, the church was partially rebuilt, as appears to be confirmed by the timber framework of the roof over the choir, which has a dendrochronological dating of 1055-1070. Heavily restored around 1708, the framework presents the technical characteristics of other Romanesque roof frameworks, with a structure of chevrons-forming-trusses, trusses with closely spaced tie-beams, assemblies at « mi-bois » and a compressive System for distributing the loads. The apse is perfectly preserved with provisional support mechanisms that indicate an error in the conception of the structure. Sculptures on the butt end of the tie beams serve as modillions at the top of the walls bearing gutters.

\section{Résumé}

L'église Saint-Pierre de Neufmarché-en-Lyons en Seine-Maritime fut le siège vers le milieu du Xle siècle d'une collégiale attachée à la famille des Grandmesnil, gouverneurs de la place de Neufmarché. Convertie en prieuré et placée sous la dépendance de l'abbaye de Saint- Evroult-en-Ouche vers 1065, l'église fut reconstruite en partie ce que semble confirmer la charpente conservée sur le chœur, datée par dendrochronologie de 1055-1070. Très remaniée vers 1708, cette charpente présente des caractéristiques propres aux techniques de charpenterie romanes avec une structure à chevronsformant-fermes, des termes à entrait rapprochées, des assemblages à mi-bois et un système de répartition des charges compressif. Elle possède notamment une croupe parfaitement conservée avec des dispositifs d'étalements provisoires qui témoignent d'une erreur de conception de la structure, et des sculptures en abouts d'entrait faisant office de modillons pour le haut des murs gouttereaux.

\section{Zusammenfassung}

Ein skulptierter Walmdachstuhl des 11 . Jahrhunderts : die Kirche Saint-Pierre von Neufmarché-enLyons, von Frédéric Epaud und Vincent Bernard

Die Kirche Saint-Pierre von Neurmarché-en-Lvons im Departement Seine-Maritime war um die Mitte des 11. Jahrhunderts der Sitz eines Kollegiatsstifts, das der Familie Grandmesnil unterstellt war, welche die Herrschaft über den Platz Neuf-marché ausübte. Als sie gegen 1165 in ein Priorat umgewandelt und der Abtei Saint-Evroult-en-Ouche unterstellt wurde, wurde die Kirche partiell neuerrichtet, was der über dem Chor erhaltene und dendrochronologisch 1055-1070 datierte Dachstuhl zu bestätigen scheint. Obwohl er 1708 sehr überarbeitet wurde, zeigt dieser Dachstuhl die den romanischen Dachstuhltechniken eigenen Merkmale mit einer Struktur von binderformenden Sparren, mit dicht stehenden Bindern mit Fragbalken, Halbholzverblattungen und ein auf Druck basierendes System der Lastenverteilung. Er besitzt insbesondere einen perfekt erhaltenen Walm mit provisorischen Stützbalkenvorrichtungen, die von einem Fehler in der Planung der Struktur zeugen, sowie Skulpturen an den Enden der Fragbalken, die als Konsolen an der Oberkante der Traufwände dienen.

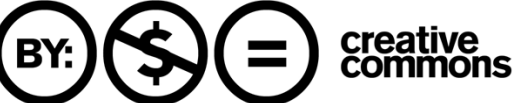




\title{
UNE CHARPENTE SCULPTÉE DU XI SIĖCLE : L'ÉGLISE SAINT-PIERRE DE NEUFMARCHÉ-EN-LYONS (SEINE-MARITIME) '
}

\author{
Frédéric ÉPAUD) et Vincent BERNARD
}

䈀 ituce sur la rive gauche de llipte. aux marges du Vexin normand, la place forte de veufmarché consti-

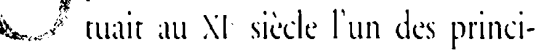
paux points de coneróle de la fronciere occidentale du duche de Normandic. Hugues de Cirandmesnil, gouverneur de cette place ex fondateur arec la famille de Giroie de labbare de Saint-Eiroult-enOuche dans l'Orne. dota en 1050 l'élise Saint-l'ierre de Veufmarché sur ses droits de péages et des moulins :

Il est probable que, des cette époque. l'église fut partagéce entre la desserte paroissiale et une communauté de clercs séculiers voués au service religieux de la famille des sires de Neufmarché. En effer, Orderic Vital mentionne quen 1065 " les quatre chanoines qui occupaient l'église Saint-Pierre s'acquittaient arec négligence du service de Dieu et vivaient trop dans le monde " . Les chanoines furent alors remplacés par des moines de l'abbaye bénédictine de Saint-Evroult-en-Ouche et la moitié du revenu de l'église fut transférée à ces moines '. C'est donc probablement à cette époque, lors de la conversion de la collégiale Saint-Pierre en prieuré, que le chour et le transept de l'église furent reconstruits. pour être affectés à cette nouvelle communauté. La nef, toujours vouée à la desserte de la paroisse, resta dans son état initial (fig. 1).

En 1128, le gouverneur de la place, Guillaume de Roumare, porta le nombre de moines à sept et fit procéder à des travaux dans les bâtiments conventuels et le chœur ". Ces derniers se sont limités au réaménagement des passages entre le chour et les chapelles latérales.
Les batiments conventucls, accolés au nord de léglise, furent reconstruits vers 1261 selon une mention rapportce par larcheréque de Rouen Eudes Rigaud lors dune de ses visites au pricuré". Un passige et un escalier furent amenagés dans le mur nord du transept, seton les recommandations de cet archeréque, pour permetere aux moines l'accès direct de leurs cellules au chorur et les isoler des paroissiens. Seul subsiste de ces batiments conventuds un pan de mur nové dans des maçonneries contemporaines er le passage du bras nord du transept.

lédifice ne subit que très peu de modifications jusqua 30 décembre 1705 lorsque le clocher se renversa sur les toitures du choeur et du transepe à la suite d'une tempéte. La restauration du clocher et de la charpente des combles ne fut acherée quien 1708 :

Enfin, dans les années 1867-1868, la nef fut en grande parcie reconstruite. Seul le mur nord conserva dans les première et dernière travées ses maçonneries romanes de silex en opus spicatum avec des vestiges de deux baies du XI' siècle.

\section{DESCRIPTION DE L'ÉDIFICE}

Le chøur, le transept ex les deux chapelles orientales présentent une homogénéité de structure, d'appareillage et d'ornementation architecturale qui traduisent une seule campagne de construction corrélative à la transformation de la collégiale en prieuré par Hugues de Grandmesnil vers 1065 (fig. 2).
Le chaur comporte une trarce droite routece en bercealu terminée par une abside couvere d'un cul-de-four (fig. 3). Il est flanqué latéralement de deux chapelles a cheret plat communicant chacune arec le choeur par un passage, réaménage au XII siecke, en arc brise retombant sur une imposte décorée d'un damier. Ces chapelles étaient couvertes à l'origine d'une voute diartes.

Les murs, percés de larges baies en plein cintre soulignées à lextérieur d'un cordon, sauf du coté des batimeress conventucls au nord, sont constitués extérieurement din blocage de rognons de silex disposés du cotré extéricur en opus spicatum dans leur parric basse er, all-dessus. d'un moven appareil de pierres calcaires. Les sculprures du bandeau fournissent une panoplie diverse de feuillages, de palmettes, de pointes de diamant et de rosettes finement trataillées.

Des contreforts plats à un seul ressiut flanq̨uent les maçonneries sur le pourtour de l'abside ainsi qu'aux angles des bras du transept et des chapelles latérales. À la naissance de l'abside, les contreforts montent jusquiau sommet des murs tandis que, sur son pourtour, ils s'arrêtent aux deux tiers de la hauteur. Le long des murs latéraux, les poussées de la voûte en berceau sont reprises par les murs-pignons du chevet plat des deux chapelles latérales.

Les bras du transept, couverts aujourd'hui d'un plafond moderne en bois, ne comportaient pas à l'origine de voûtement en pierre, mais un couvrement en bois sous forme, vraisemblablement, d'un plafond fixé en entrevous des entraits de la charpente. 


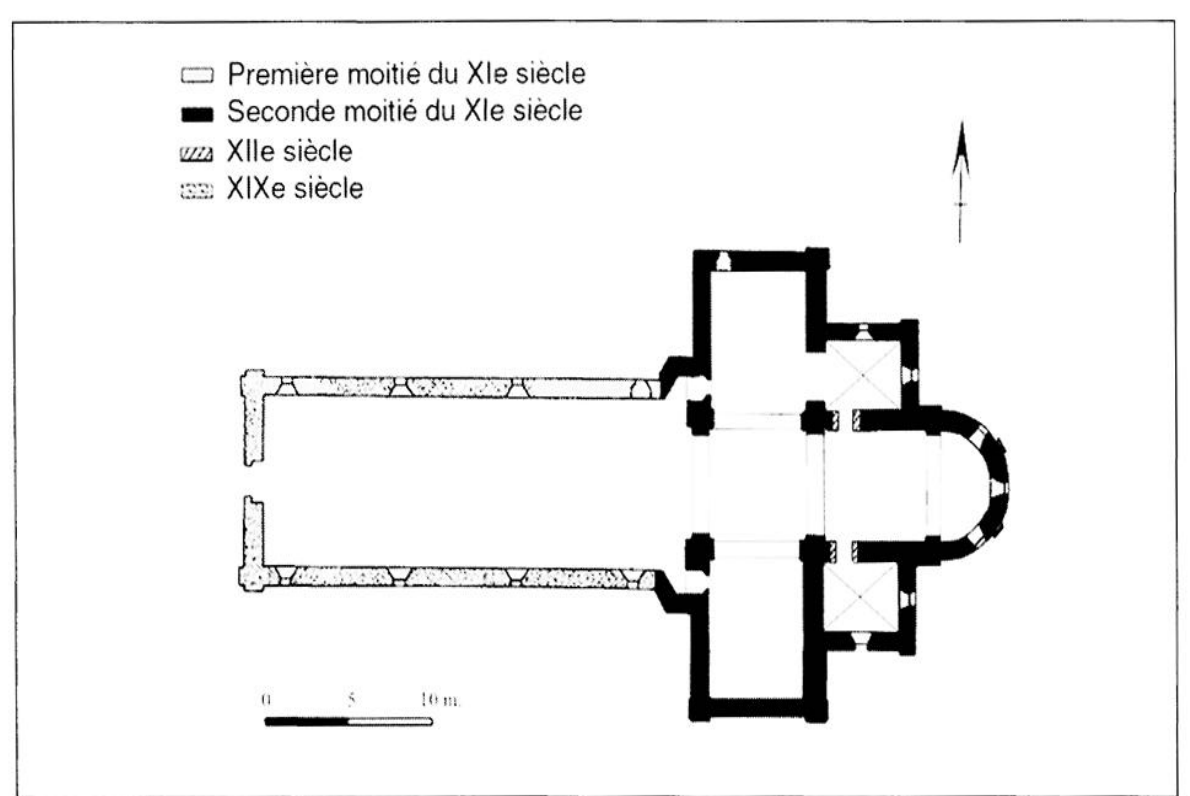

Fig. 1 - Plan au sol de l'eglise de Neufmarché-en-lyons, diapres Ruprich-Robert, l.Architecture normande, t. I pl. IX, fig. 4.

L'édifice se distingue par des murs gouttereaux d'une très grande hauteur, une sobriété ornementale marquée par la rarcté des sculpures, limitées à l'extérieur, l'absence de chapiteaux. ainsi que par quelques dispositions remarquables dans la Normandie romane, comme les passages au revers des piliers ouest de la croisée et la voûte en berceau couvrant le choeur. Néanmoins le dépouillement des élévations, l'austérité de l'ornementation et la rigueur de l'ensemble répondent bien aux normes décoratives des constructions normandes du temps de Guillaume le Conquérant.

Il subsiste, exposés dans le chour, quelques éléments sculptés de l'ancien portail occidental roman démonté au siècle dernier et qui, selon Lucien Musser, comportair une porte principale flanquée de colonnettes portant un trmpan et de deux petits portails latéraux datant du second quart du XII siècle ". Ce portail pourrait donc se rattacher, comme les passages latéraux du choeur, aux travaux entrepris en 1128 par Guillaume de Roumare. niées. La charpente voùtéc et lambrissée de la nef semble appartenir à la fin du Moren Âge, tandis que la charpente du clocher ainsi que les combles du transept ont éré refaits en 1708, après l'effondrement du clocher, le 30 décembre $1705^{\prime \prime}$.

Le comble du choeur, dissimulé par la voûte romane, comprend de nombreux éléments d'origine, encore en place malgré les travaux de restauration de 1708 . Il se compose d'un ensemble de huit fermes disposées sur la travée droite du chocur, longue de $5,23 \mathrm{~m}$., er d'une croupe ronde assise sur l'abside. Les analyses dendrochronologiques menées sur les bois de cette charpente indiquent une mise en place dans les années 1055-1070. I.a construction de l'église pourrait donc coïncider avec l'ćrection du prieuré, en 1065.

\section{DISPOSITION ACTUELLE DES FERMES} (fig. 4)

La structure actuelle, issue des remaniements de 1708, est composée de cinq fermes constituées chacune de deux chevrons, de deux jambes de force et d'un entrait à la base, de deux fermes à portique supportant un cours de pannes sur chaque versant et enfin d'une ferme de croupe, à l'est, distincte des précédentes (fig. 5 et 6). Lespacement de ces fermes en entrevous des entraits est de $54 \mathrm{~cm}$ en movenne.

Une panne-faitière assemblée en retc du poinçon de la ferme de croupe et fixée à l'autre bout dans les maçonneries de la tour, comme les pannes, supporte la tete de ce chevronnage. Deux liens obliques

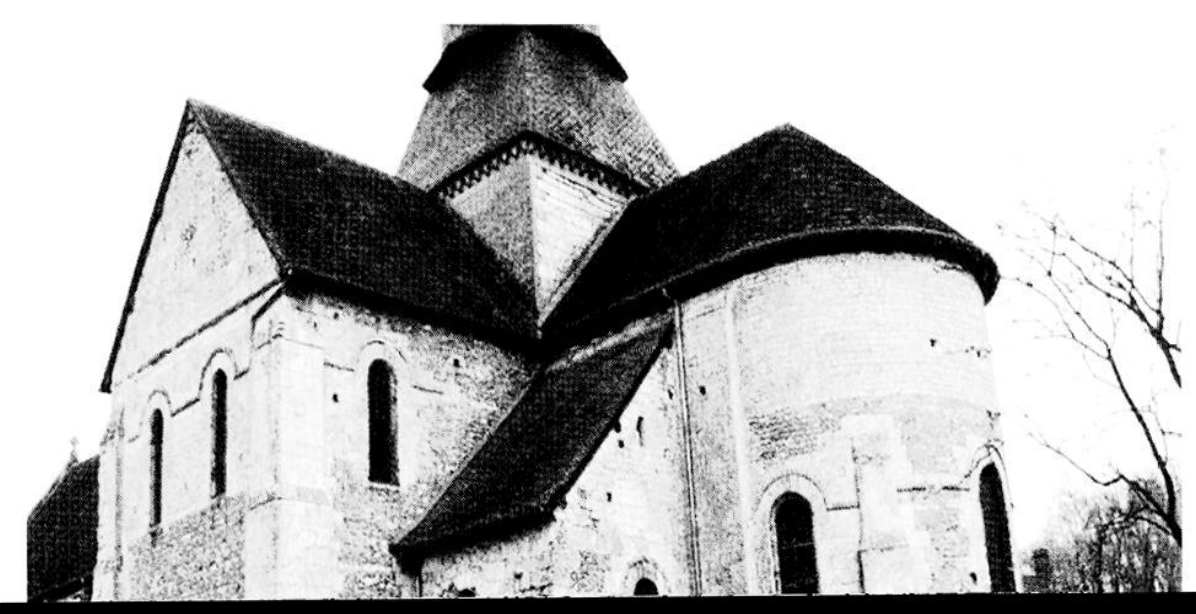




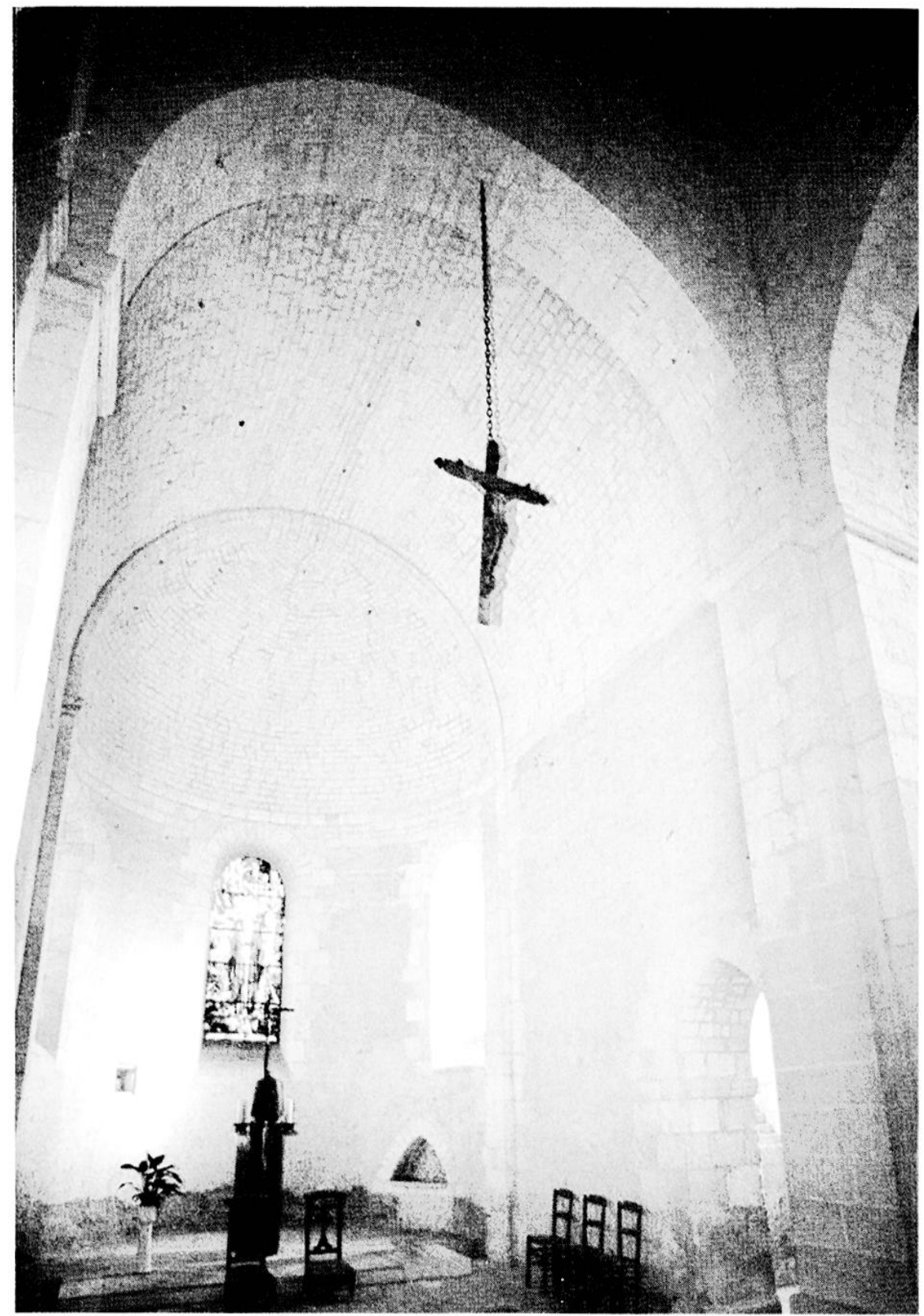

Fig. 3 - Vuc intérieure du chơur.

assurent le contreventement longitudinal de cette panne-faitière et donc de l'ensemble de la charpente.

Les deux fermes à portique, de composition moderne, sont constituées chacune d'un couple de chevrons soutenus par deux jambes de force, d'un entrait à leur base et d'un portique. Destiné à soutenir les pannes, ce portique se présente sous la forme de deux montants obliques sur la tête desquels vient s'assembler une pièce horizontale, le chapeau. Les pannes reposent sur des échantignoles tenonnées et chevillées sur le flanc de ces montants obliques.
Tous les entraits présentent des entailles vides d'assemblagess à mi-bois. cn queue d'aronde ou en demi-queue d'aronde, ainsi que des signes évidents d'un remaniement important, tels des amputations aux extrémités et des greffes. On constate que les entraits des fermes 5, 6,7 et 8 , par la position et la symétric de leurs entailles à mi-bois, sont en place et que les autres entraits ont éré déplacés pour subir une greffe sur une partie de leur longueur.

Les éléments du chevronnage sont de trois types. Le premier concerne des bois équarris a la scic, sans assemblage et de faible scction, mis en place vraisemblablement en 1708 et qui sone présents sur le versane nord et dans l'enerevous des cherons des fermes sur les deux versants. Ie deuxieme type se rapporte aux cherrons des fermes 1, 2, 3 et 4, équarris également a la scic, mais qui présentent des traces d'anciens assemblages, tcls un cmbrevemene en pied pour une jambe de force ou un mi-bois en demi-guecue d'aronde au tiers de la hauteur et trois trous de chevilles sur le restant de la longueur. destinés a la fixation de pannes. (és cléments correspondent à des rémplois provenane dunce autre charpente, peut-eitre des bras du transept, refaites en $1^{-} 08$. Enfin, ke troisième rype sapplique aux cherrons sud des fermes 5 is 8 et aux deux entre-cherons sud des fermes 2-3 et 3-4 qui presentent chacun trois entailles d'assemblages a mibois sur la face est. régulierement espacies. ainsi yu un mi-bois en tite du cherron. La correspondance de ces assemblages arec ceux des entraits et l'équarrissage des bois à la deloire confirment lappartenance de ces derniers cherrons à la structure d'origine.

Remaryuons également les nombreux éléments de réemploi, reconnaissables aux entailles à mi-bois non utiliscées, dans le porrique des fermes et parmi les jambes de force.

La ferme de croupe se distingue des fermes précédentes par une disposition complètement différente de ses éléments constitutifs (fig. 6). Un couple de chevrons, pourvus chacun diune jambe de force, prend appui en pied aux abouts d'un entrait et en tête sur les flancs d'un poinçon de fond. Chaque cheuron est raidi par un poreau vertical, assemblé à ses extrémités par un mi-bois, et par un faux entrait assemblé également par mi-bois aux chevrons, aux poteaux et au poinçon. La moirié nord de ce faux entrait a été remplacée tardivement et le pied de ces deux poteaux a été déchaussé.

Lassemblage à mi-bois de ce faux entrait au chevron sud présente une particularité qui mérite d'être relevée (fig. 7). 


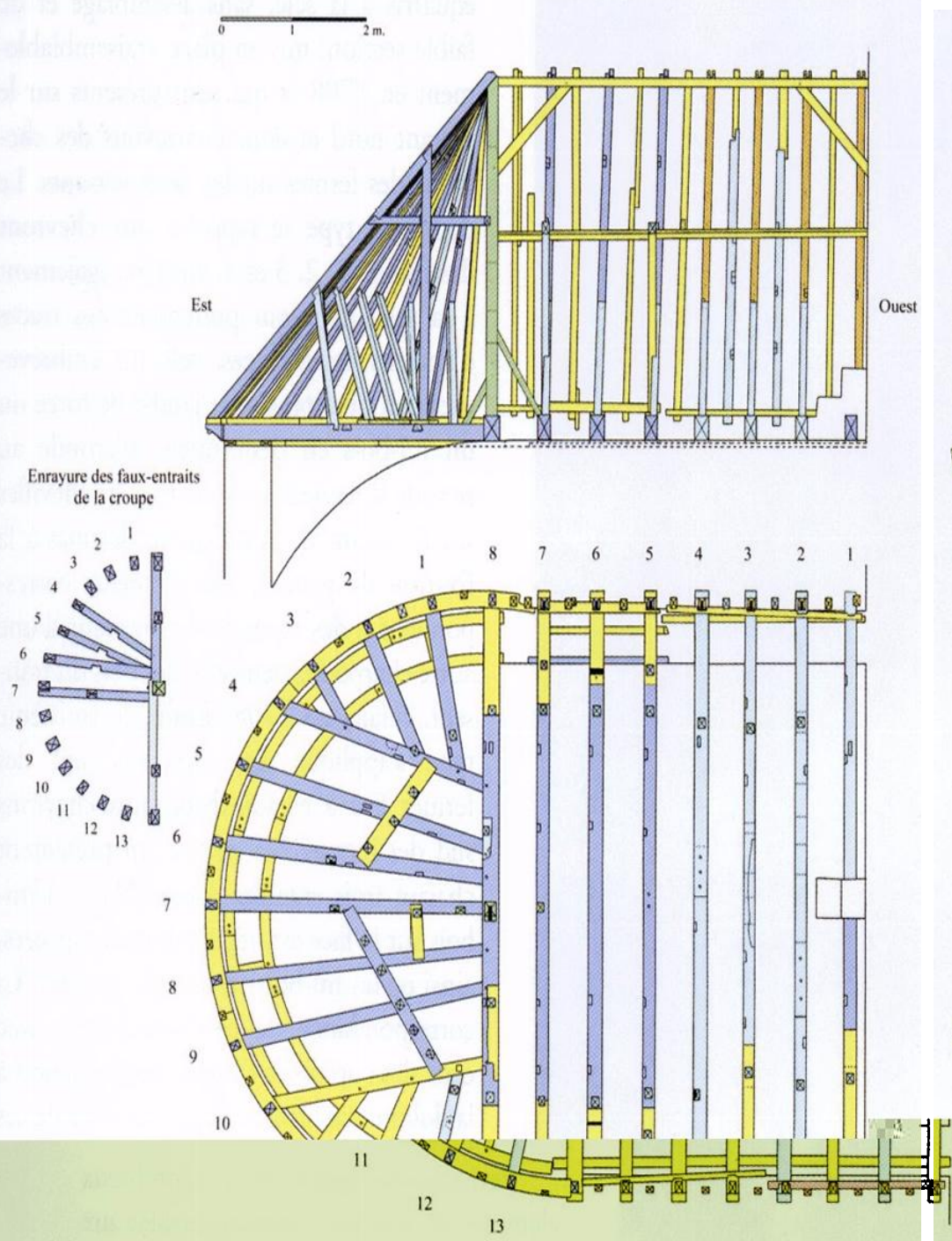

Fig. 4 - Coupe longitudinale et plan de la charpente du chœur de l'église Saint-Pierre de Neufmarché.
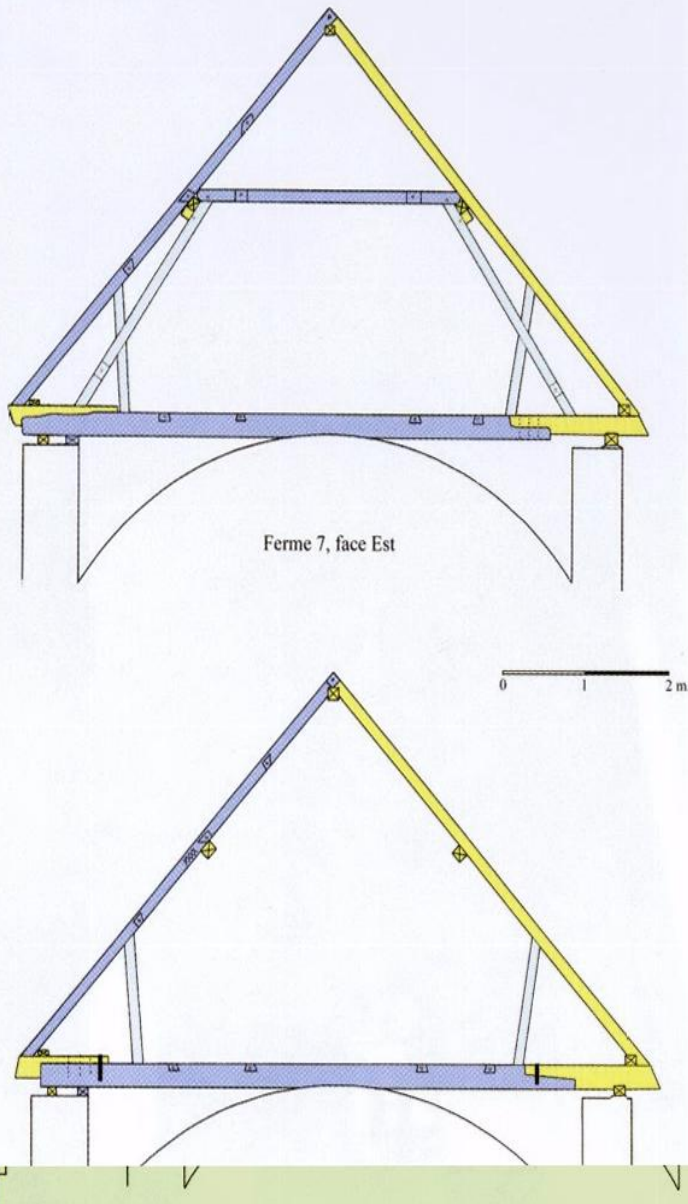

Fig. 5 - Fermes actuelles.

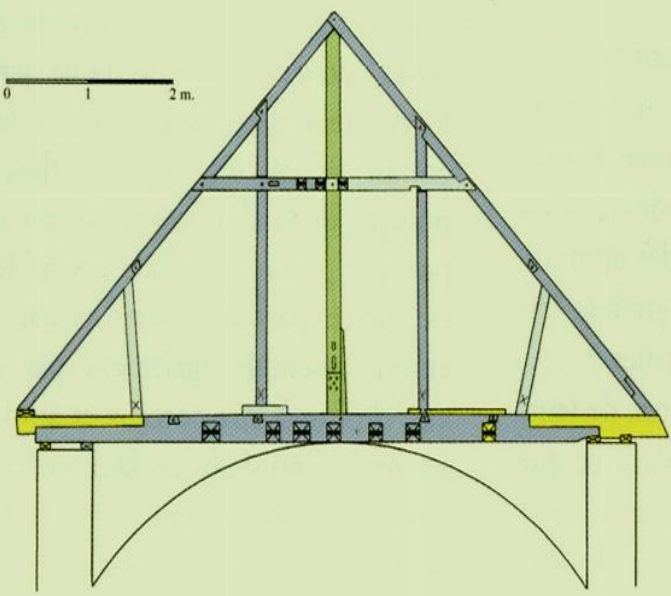

Relevé et dessin F. Épaud

1055-1070, bois en place ou réempoyé dans sa position d'origine

$\square$ 1055-1070, bois déplacé

Fin Xle - début Xlle siècle

$\square 1708$

Bois d'origine inconnue réemployé en 1708

XXe siècle

Fig. 6 - Ferme de croupe, F8 face est. 


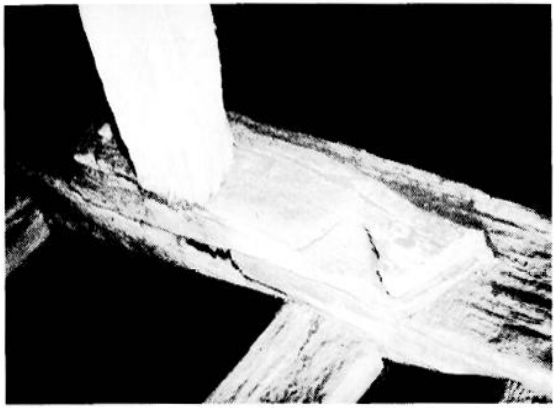

Fig. - Assemblage diabout de Pentrait retrousse de la leme de croupe rimplosic en scmelle c'n T'0s.

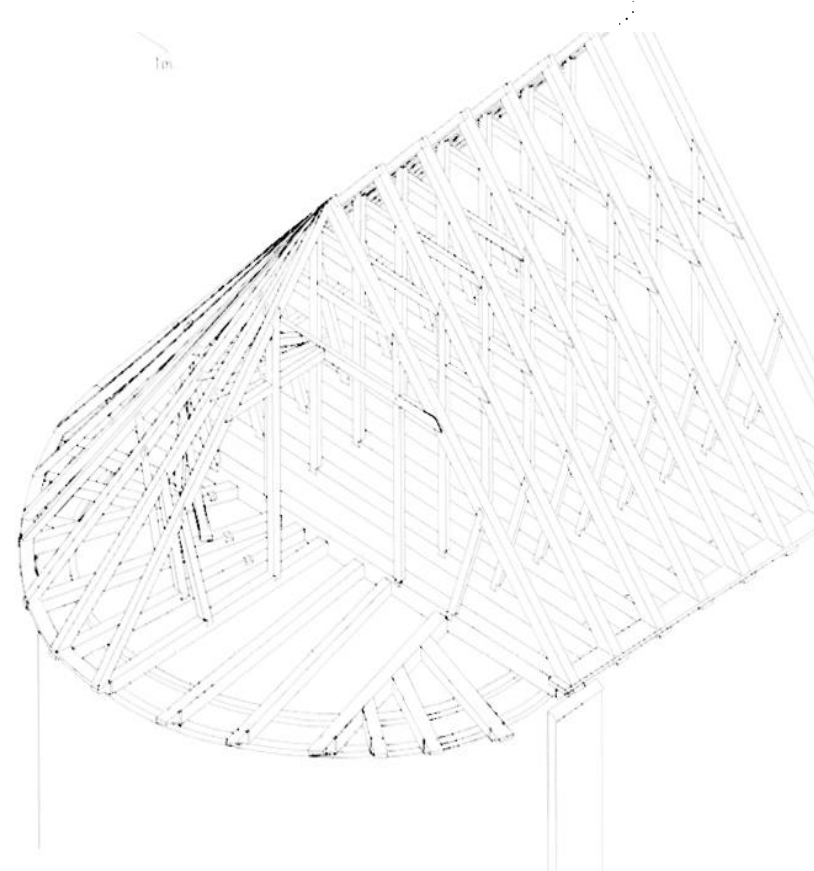

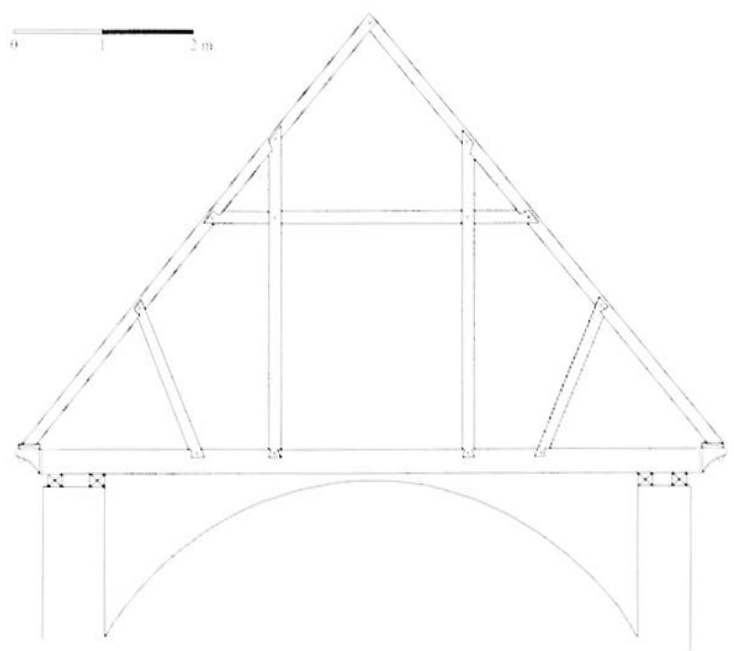

Fig. 8 - Resceution d unc forme derigine (dessin l: Fpaud).

Fïg. 9 - Resriturion axonomérricue de la charpente (releve er dessin 1: Fpaud).
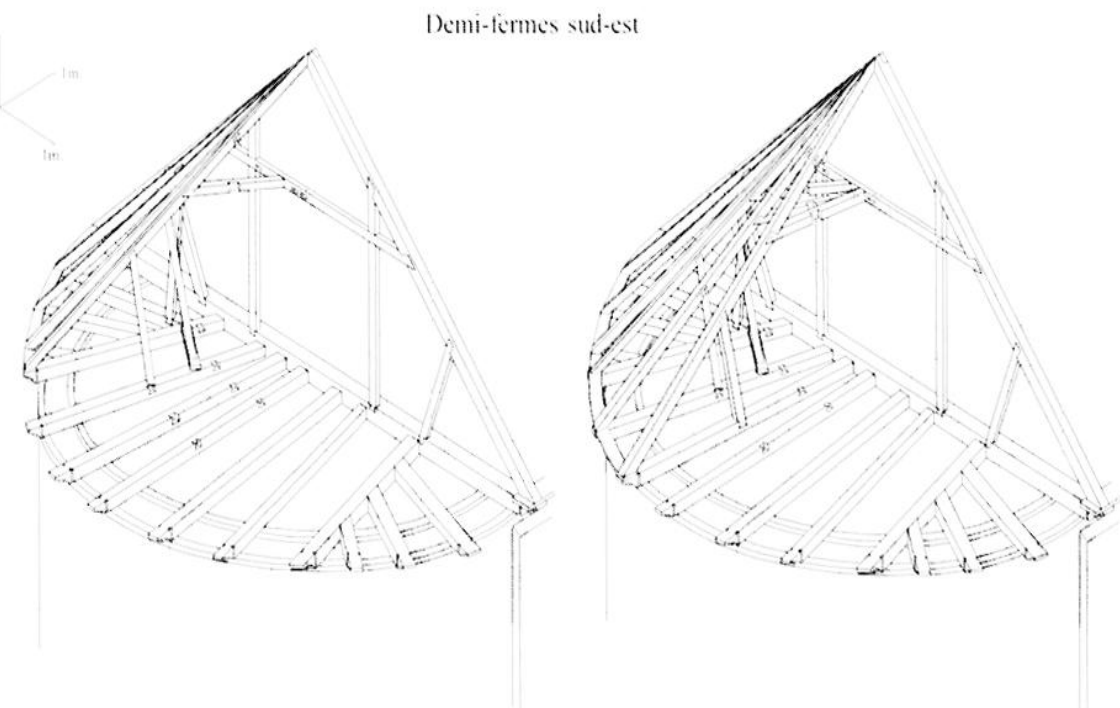

Fyalise de Neufmarche-cin-lyon

Hig. 10 - Vues axonométriques de la croupe (relevé et dessin F. Épaud).

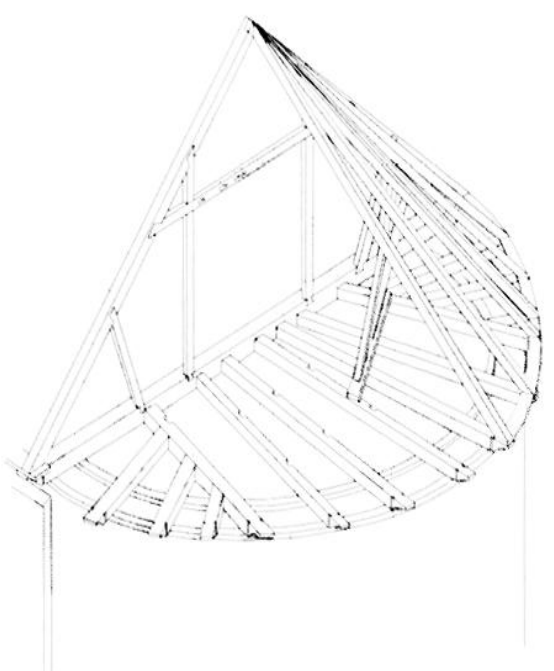




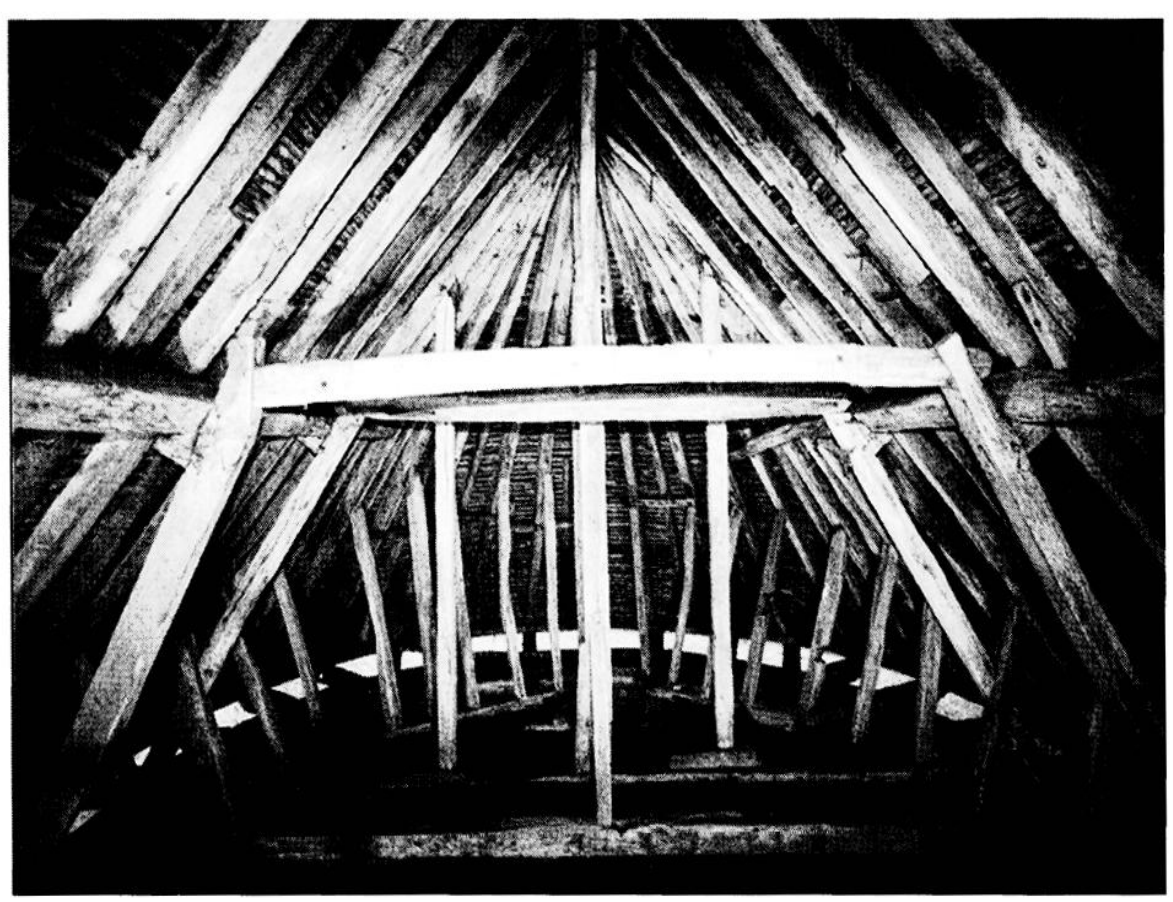

Fig. 11 - Vue de la croupe.

Ce faux entrait a été mis en place en applique dans les entailles à mi-bois pratiquées dans le poinçon, les poteaux et les chevrons sur leur face est. Bien quayant une section identique à ces bois, voisine de $10 / 11 \mathrm{~cm}$, le faux entrait n'a pas été assemblé à mi-bois mais au ciers de sa largeur, laissant ainsi une surépaisseur de bois qui recourre les assemblages et les dissimule à la vue. Cetre particularité s'explique par le fait que cette pièce reçoit les faux entraits des demi-fermes de la croupe er que des assemblages normalement taillés à mibois, sans renfort, auraient logiquement cédé sous les charges. Ces assembliages à tiers-bois sollicitent moins de fibres ligneuses et autorisent donc plus de percements d'assemblages, notamment ceux pour les faux entraits des demi-fermes. Cette précaution dans la taille des mi-bois sobserve également sur les bois romans réemployés au début du XIII siècle dans la nef de la carhédrale de Rouen, bois qui nont éré entaillćs quau tiers de leur section, en raison des nombreuses entailles à mi-bois non réemployées qui fragilisaient ces pièces.

Les entraits de ces fermes sont portés au nord par une seule sablic̀re monoxyle sur toute la longueur de la charpente et, au sud, par une sablière pour les fermes 1 à 4 et par deux sablières pour les fermes 5 à 7 . Seule la sablière interne de ces deux dernières semble d'origine. Il sagit d'une pièce extrêmement endommagée par l'érosion, réduite à une section de $10 / 12 \mathrm{~cm}$, mais qui présentait à l'origine une largeur de $17 \mathrm{~cm}$ daprès les traces laissées en négatif dans le mortier de pose au sommet du mur roman. Laltération des sablières d'origine et leur remplacement permetrent d'expliquer en partie l'affaissement du niveau des entraits et le fait quilils reposent aujourd'hui sur l'extrados de la voûte.

\section{RESTITUTION DES FERMES D'ORIGINE}

La disposition des éléments de la ferme 8 semble correspondre, d'après la distribution des entailles à mi-bois sur les chevrons et les entraits réemployés des autres fermes, à la structure primitive. La position des mi-bois sur les bois de réemploi et l'inclinaison des gorges de leurs entailles concordent précisément avec le schéma structurel de cette ferme.

Cette charpente se composait donc de huit fermes strictement identiques, indépendantes les unes des autres, sans aucun dispositif d'étrésillonnement ou de contreventement longitudinal (fig. 8 et 9). D'une portée d'entrait de $5,94 \mathrm{~m}$, ces fermes avaient un entraxe moven de $72 \mathrm{~cm}$ et une inclinaison des chevrons de l'ordre de $47^{\circ}$.

Chaque ferme possédait à la base un entrait de $26 \mathrm{~cm}$ de haut sur $17 \mathrm{~cm}$ de large en moyenne, qui reposait sur les murs gouttereaux par l'intermédiaire de deux cours de sablières, sans assemblage. Un couple de chevrons, assemblés en tête par mi-bois, était raidi par un faux entrait, deux poteaux verticaux et deux jambes de force obliques. Tous ces éléments sassemblaient sur la face est par mi-bois à demiqueue d'aronde aux cherrons et à l'centrait, et par mi-bois à queue d'aronde pour le pied des poteaux. De même, le faux entrait s'encastrait par mi-bois sur la face est dans les poteaux. Aucun assemblage à tenonmortaise n'entrait donc dans la composition de ces fermes. Les poteaux, les faux entrairs, les chevrons et les jambes de force présentent une section particulièrement faible, de l'ordre de $10 / 11 \mathrm{~cm}$. Aucune rainure ni aucun autre dispositif de fixation d'un plancher n'ont été relevés sur les quatre faces des entraits, ce qui prouve que ces derniers n'ont jamais été apparents et que la mise en place de la charpente est bien contemporaine de la construction de la voûte.

Ce type de fermes dorées de poteaux verticaux assemblés à un faux-entrait, avec un entrait à la base, est attesté pour la période romane en France septentrionale. On connait les charpentes des églises Saint-Germain-des-Prés (ca 1018-1038) et Saint-Pierre-de-Montmartre (11641174 d) à Paris ", celles de l'église de Merlemont dans l'Oise ': er de l'église de Chivy-lès-Ftouvelles dans l'Aisne (11291139 d) $\because$. Dans ces derniers cas, les entraits servaient de support à un plafond apparent constitué de planches alors quïci. comme pour la charpente romane réemplovée de la cathédrale de Rouen conscituée de fermes à entrait, la charpente était dissimulée par les voûtes. La présence d'un 
enerait a la base de chacyue ferme nest done pas inherente a lexistence diun platond mais constituc réllement un dément typologiegue de la structure de ces charpentes romanes.

Ce tope de charpente à cherrons-formant-fermes consticue la forme la plus archä̈puc recensice a lheure actuelle pour le nord de la France. Il se définit par un sisteme de répartition des charges répondane uniquement a des efforts de compression et de flexion, par une absence de contreventement, unc pente faible, inférieure a 5()$^{\prime \prime}$, et par lemploi exclusif de lassemblage a mi-bois. notamment en pied de piecess. less deftauss de certe répartition des charges sont compensés part la réduction au minimum de la section des clements qui transmettent les forces de compression (cherrons, poteaux, jambes de force... er par l'augmentation cecessive de lépaisseur des contrairs qui les reçoivent et traraillent done en flexion. La taible pente du cherronnage participe aussi a la reduction des charges compressives en limitant la surface de la toirure et done le poids des matériaux de couverture.

Toutes les fermes étant identiques et rapprochées, les charges de la charpente se répartissent de façon homogiene et uniforme sur les murs gouttereaux. Ce type de charpente exige done des murs résistants qui se doivent d’ètre épais sur toute leur longueur. Cette structure devait rapidement disparaitre dans le courant du xill siècle avec l'apparition de la travée au sein de la charpente, mieux adaprée à l'évolution des supports maçonnés qui tendent à samincir, à souvrir de plus grandes baies et à répartir les charges du couvrement et du vouttement selon une trame articulcé sur des contreforts de plus en plus saillants. Parallèlement, la constitution des fermes va évoluer vers une meilleure maitrise du raidissement des chevrons, qui aura tendance is supprimer les éléments verticaux de transmission des charges et à libérer ainsi les entraits du report de ces charges.
LA CROUPE (fig. 9)

La majeure partic des éléments de cecte croupe nia subi que trés peu de modifications ex se troure toujours en place. Fille comprend une ferme de croupe er 1.3 demi-fermes constituces chacune d'un cherron, étaré par une contrefiche, er d'un demi-entrait a leur baste (fig. 10). Un cours de sablieres cherillées sur l'extrémité des demi-entraits sur tout le pourtour de la croupe receptionne dans une perite cavité ke pied des chererons at des encre-cherrons : Lenrayure des 1.3 demi-entraits sorganise selon un plan raronnant arec sepe demi-contraits médians, assembles à lentrait de la ferme 8 par tenon-mortaise. complétés par six embranchements répartis au nord et au sud de la croupe.

l.es cherrons de la moitic nord die la croupe sone écarés par une contrefiche dont la rete, grossierement dégrossie, vient sencastrer dans une carvite en forme de morraise sans cherillage et dont le pied sappuic sur unc piecce de bois posice a plat sur plusicurs entraits (fig. 11). Dans la moitic sud de la croupe, les demi-termes sone exécurées, a l'exception des demifermes 2 er 3 . comme une moitic de ferme principale, arec un demi faus-entrait et des entailles d'assemblages à mi-bois sur le cherron et le demi-entrait pour receroir une jambe de force er un poteau vercical. ()n remarque cependant quaucun potcau ni jambe de force na a pu être mis en place dans ces assemblages puisque le plan verrical des cherrons ne correspond pas à celui de leur demi-entrait. Seule la demi-ferme 7. placée dans l'axe de la croupe er dont le plan vertical du chevron concorde avec celui de son demi-entrait, présente cette disposition de pièces a l'image d'une demi ferme principale. La demi-ferme 6 a pu seulement assembler sa jambe de force en pied dans le demi-entrait, mais avec une forte inclinaison pour rattraper l'écart du chevron. Faute de poteaux, les autres chevrons sud sont étayés par une contrefiche assemblée par un mi-bois ou dans une carité, et appurece sur une sole exactement comme au nord.

(ies assemblages vides timoignent indéniablement d'une crreur de conception commise lors de lexécurion au sol des demi-fermes. tilles ont, en ciffer, cité exicutées sur lépure des fermes principales. l'espacement de ces assemblages étant strictement identique à celui des fermes principales . la croupe a donc été conçue comme unc structure pourant se composer de demi-fermes reproduisant exactement le schima d'une moitie de ferme principale et disposces seton un plan rayonnant. Cependant, limpossibilité rechnique de faire correspondre lentarure des demi-enerairs arece celle des demi-taux. encrails dans le mime plan verrical des cherrons a contraint les charpentiers a improviser un dispositif déraiement pour remplacer les poteaux en disposant sur une sole, répartic en travers sur les entraits, des contrefiches assemblées aux cherrons par mi-bois ou par encastrement.

les demi-fermes nord ne présentent pas dentailles vides de mi-bois et semblent aroir été conçues et réaliscés spécifiquement pour es systeme d'étaiement par contrefiches en appui sur les encraits. $\mathrm{Ne}_{\mathrm{c}}$ reproduisant pas cee défaut de conception, les demi-fermes nord semblent donc aroir été exécutées après les demi-fermes sud de lia croupe, apres que les charpentiers prirent conscience de leur erreur.

Les demi-faux-entraits des demi-fermes sud, non raidis par les porcaux inicialement prévus, ont reporté toutes leurs charges sur le faux entrait de la ferme de croupe auquel ils sont assemblés par tenon-mortaise. Ce dernier a donc fléchi sous cette surcharge, ce qui a contraint les charpentiers dans un second temps à mettre en place au sein de la ferme un poinçon assemblé par un mibois à ce dernier de façon de le soulager. Cette réparation, datée par dendrochronologie du dernier quart du Xl' ou du premier quart du XII siècle, est attestéc par le fait que le poinçon n'a pas été assemblé en tête aux chevrons et quiil est composé de deux 
pièces assemblées par un mi-bois "pour être inséré en sous-øeuvre dans la ferme. Ce poinçon travaille donc uniquement en compression, sans triangulation, comme un poteau.

Lerreur de conception commise sur les demi-fermes sud et cette réparation consécutive résultent d'une façon de concevoir les charpentes de combles selon sur une perception bi-dimensionnelle de la structure et donc de la difficulté pour des charpentiers du Xl siècle à projeter et à exécuter au sol une structure assembléc en trois dimensions. La plupart des charpentes de comble de cette époque sont des structures constituées de fermes indépendantes dépourvues d'ććments longitudinaux et aucune autre croupe du Xl siècle, dans l'état actuel de nos connaissances,n'a été conservée. Il faut attendre la fin du XII. siècle pour quiapparaissent en Normandie les premiers éléments longitudinaux et que se développe la conceptualisation de structures tridimensionnelles plus complexes. Le beffroi de la fin du XII siècle de la cathédrale de Bayeux présente encore une structure composée uniquement de pans de bois plus ou moins indépendants les uns des autres et non d'éléments travaillés dans trois plans directionnels.

La disparition des charpentes du Xl' siècle résulte cerres de l'évolution des formes des combles et des techniques de charpenterie aux XII-X'XII' siècles, mais elle sexplique aussi en grande partie par leurs nombreux défauts de conception liés à cette perception bi-dimensionnelle des structures de combles qui a engendré le déversement quasi systématique des fermes, lui-même facilité par des assemblages et des sections de pièces inadaptées aux contraintes de la charpente.

\section{DATATION DENDROCHRONOLOGIQUE DE LA CHARPENTE}

L'étude dendrochronologique a porté sur 21 échantillons de bois de chène (Quercus sp.) provenant de certe charpente de croupe. Bien sûr, compte tenu du caractère exceptionnel que confere la présence d'une telle structure en place sur un bâtiment roman, la priorité de cette analyse a été accordée au calage chronologique des éléments les plus anciens. 20 prélèvements correspondent ainsi a cette phase de construction ; l'échantillon restant, qui appartient probablement à la phase de restauration du XVIII s., n'a malheureusement pas pu être daté en raison d'une série de cernes trop courte.

Cette présentation d'une des rares superstructures en bois datée du milieu du XI siècle au nord de la Loire s'attachera donc à analyser les résultats de datation et à en critiquer la validité.

Làge moyen des séquences individuelles mesurées est de 58 ans et un seul échantillon, le $n^{0} 15$, présente plus de 80 cernes. Or, un seuil statistique de 80 ans limite l'urilisation du calcul dendrochronologique standard européen : seuil en deçà duquel les résultats de daration nione rien de significatif. Dès lors. le recours au test " $W$ " dans la phase de mise en place de la movenne dendrochronologique locale sest limité au contröle de position des séries les plus longues. Les corrélations staristiques, inefficaces dans le cas de Neufmarché, ont donc ćté délaissées au profit de montages visuels opérés sur table lumineuse (fig. 12). Toutctois, il faut reconnaitre que cette étape de montage se serait avérée périlleuse sans la présence, dans plusieurs échantillons, de périodes d'accroissements très faibles marquant des années particulièrement mauvaises pour l'ensemble des arbres exploités ". Ces repères méréorologiques ont donc permis d'interdater les différentes sérics de cernes.

Cette procédure termince, une séquence moyenne ("Neufmarché M11 ") longue de 1.31 ans et regroupant 15 individus a pu être constituée. Cette chronologie exprime ainsi la synthese de la croissance des chênes employés au cours de la principale campagne de construction de la charpente de croupc. Confrontée aux références dendrochronologiques européennes les plus pertinentes chronologiquement et géographiquement, la séquence issue de Neufmarché obtient une bonne proposition de datation tant du point de vue régional qu'extra régional : ainsi, le ler cerne de cette moyenne de 131 ans est situé en 957 et le dernier cerne mesuré en 1087 ", gràce notamment aux chronologies normandes provenant de Rouen, "Place de la Pucelle " (763-1070) ou de Brionne, "Le Donjon " (853-1127), mais aussi grace aux étalons dendrochronologiques constitués dans le Bassin parisien $(-348 / 1995)$ "", dans l'Est de la France (581-1991) : ou encore en Allemagne de l'Ouest (822-1964) $\because$. Le bon résultat du test "W " n'est pas le seul critère recherché ici : en effer, la réitération d'une même proposition entre plusieurs références constitue véritablement une aide dans le choix d'une datation. Son acceptation n'est, bien sûr, opérée là encore qu'après vérification sur table lumineuse $\mathrm{du}$ bon niveau de concordance entre la courbe à dater et les courbes de référence (fig. 13). En l'occurrence, la datation de "Neufmarché M11 " en 957-1087 peut être considérée comme valide, compte tenu du bon étalonnage dendrochro- nologique dont on dispose pour la Normandie.

La présence d'aubiers, et à plus forte raison de cambium (assise génératrice de l'arbre dont l'observation atteste de la présence du dernier cerne), est indispensable pour permettre datrribuer à chaque bois une phase d'abattage. Il semblc important ici, avant d'aborder les principales étapes de mise en cuvre de la charpente, de rappeler quil est admis par les dendrochronologues et par les archéologues du bàti, qu'un court laps de temps sépare généralement l'abattage de l'emploi d'arbres dans une construction $":$.

Parmi les 15 échantillons datés provenant d'arbres équarris :

- aucun ne posside de cambium ou l'un des 3 derniers cernes produits avant 


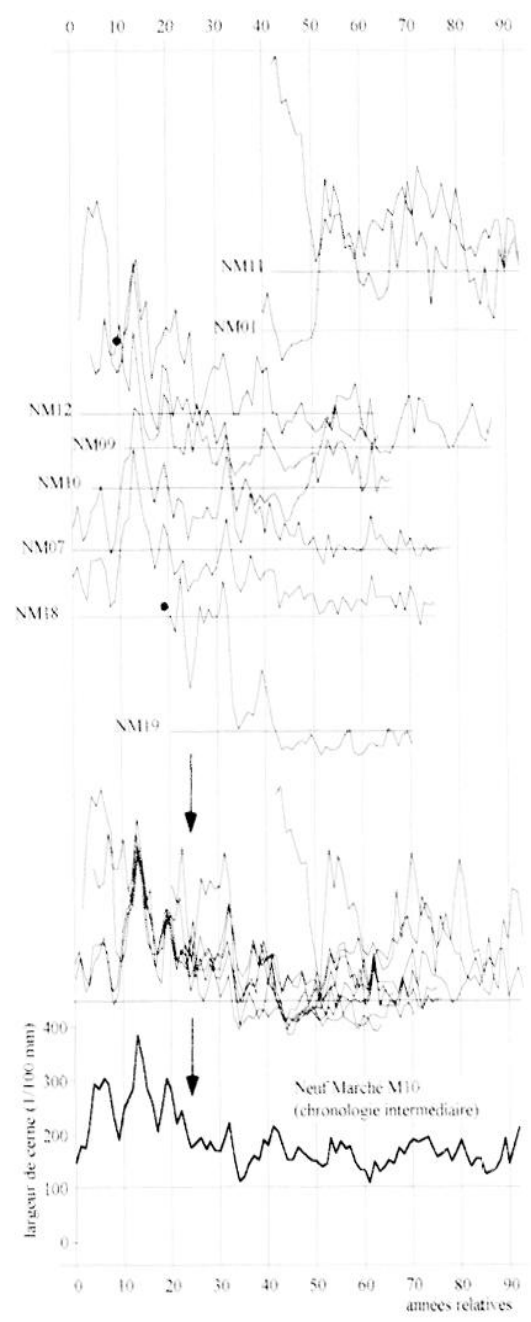

Fig. 12 - Procidure de montage de la síguence movenne " Neufmarche .110 ". depuis la sinchronisarion des séries individuclles juscua ia la conscitution de la morenne.

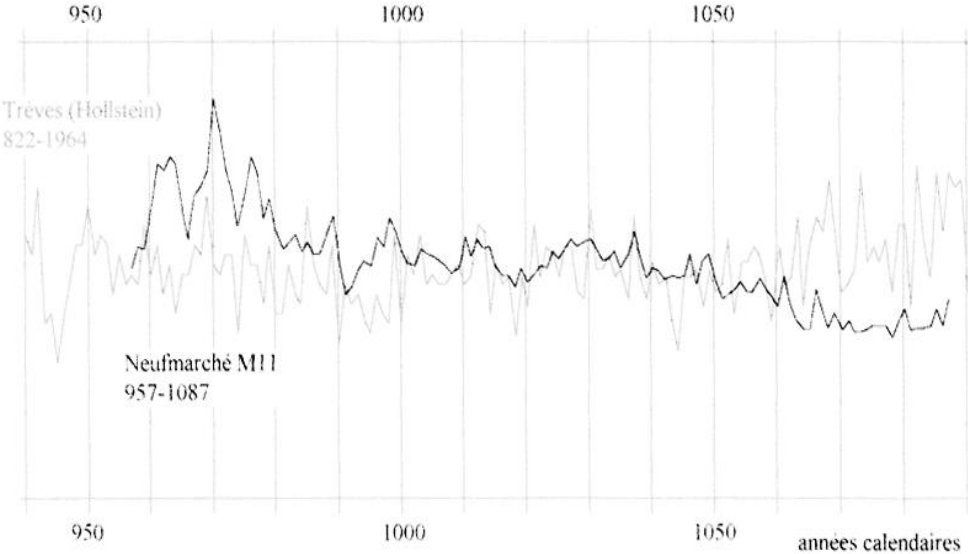

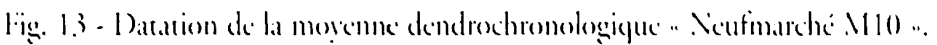

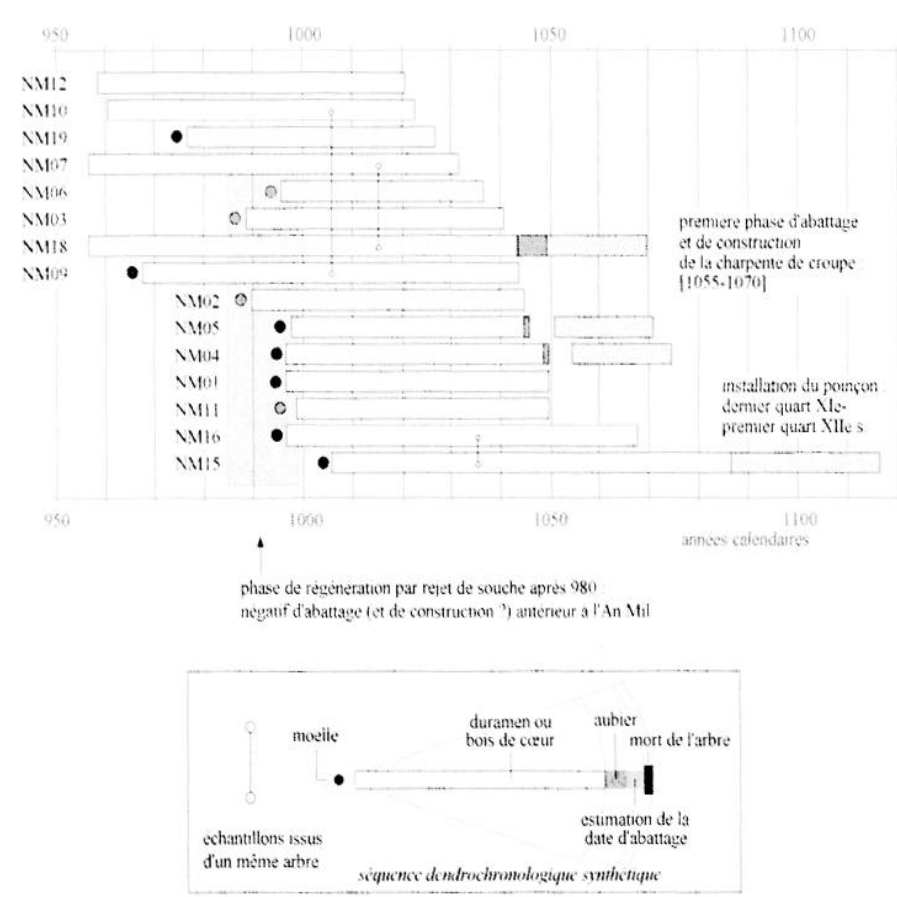

Fïg. 14 - Bloc-diagramme représentane de façon schémarique chaque siquence individuelle dans sa posirion redative au sein de la movenne "Neufmarche .1110".

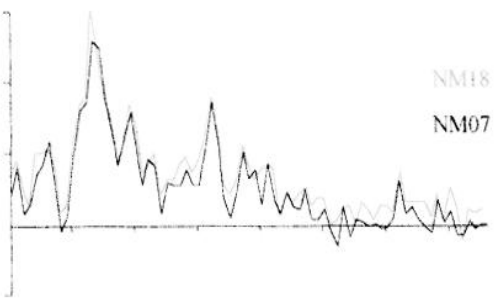

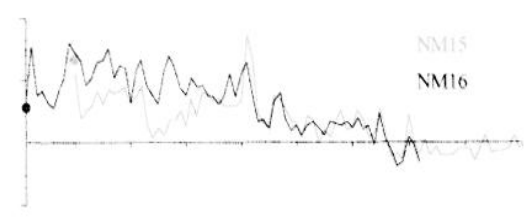

Fig. 15 - Regroupements typologiques d'échantillons provenant manifestement d'un méme arbre. 


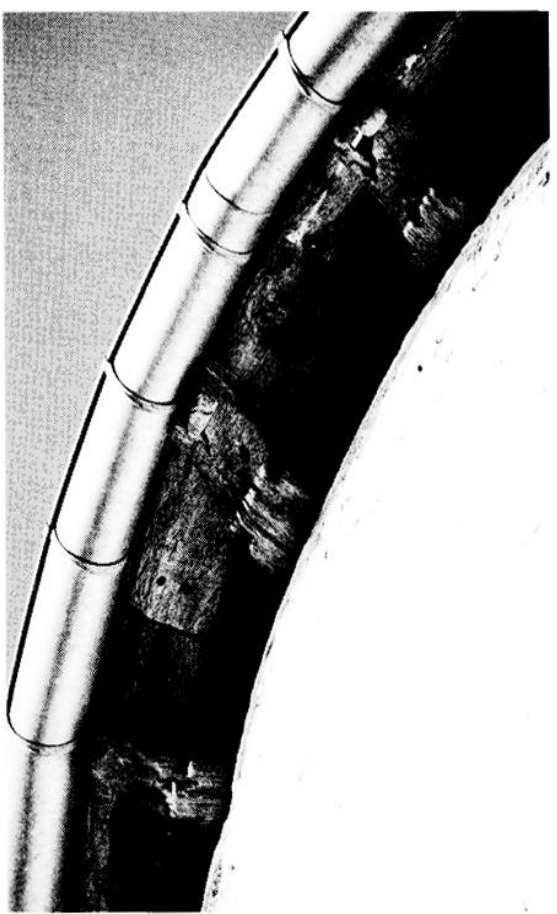

Fïg. 16-Vue extéricure des abouts dentraits de la croupe.

l'abattage. Cette caractéristique expliquera les fourchertes de datation qui seront avancées.

- Toutefois, le demi-entrait de la ferme 9 (NM18) possede un reliquat de 6 cernes d'aubier ; en ce qui concerne les demientraits 1 et 2 (NM04 et 05), leur aubier rest plus visible quà l'état de trace. Gràce à un écart-type fixant, pour la France, le nombre moven de cernes d'aubier du chêne à feuilles caduques dans une fourchette de 3 à 40 ans " (Lambert 1996), il est possible de préciser l'abattage de ces arbres dans une fourchette d'une quarantaine d'années. Cette fourchette peut être encore réduite, puisque pour la Normandie médiévale, ce nombre moyen de cernes d'aubier se situe entre 6 et 26 ans $:$.

- 14 pièces ne sont donc plus représentées que par le bois de corur (ou dwamen). Cette mauvaise conservation des aubiers peur sexpliquer par le fait que cette partie de bois à la périphérie du tronc, la plus fragile, est aussi la plus exposée aux intempérics et aux attaques d'insectes xylophages. La qualité de l'iquarrissage est assez. varriable et ne semble pas entrer ici en ligne de compte.
L'attribution à une phase d'abattage de ces échantillons sans aubier comporte donc toute l'imprécision d'un termizus post quem. En comparant ces arbres au prélèvement $n^{\circ} 18$, on parvient néanmoins à rattacher ces éléments à une phase de construction plus précise.

La phase de construction la plus ancienne mise en évidence par la dendrochronologie pour la charpente de croupe se situe, en l'absence d'aubier complet, entre 1055 et 1070 . Cette phase regroupe 13 des 15 échantillons datés, ce qui tendrait à démontrer que l'essentiel des bois encore visibles appartient à la période romane, mème si de nombreuses traces de réaménagements sont pcrceptibles.

Cetre datation de l'édification de la charpente et de l'extension du bâtiment initial au début de la seconde moitié du XI siècle coïnciderait avec la reconversion de la collégiale en prieuré, citée en 1065 dans les textes.

Une reprise médiévale de la superstructure en bois est marquice dans le dernier quarr du XI ou dans le premier quart du XII siècle par l'installation du poinçon bipartite de la ferme 8 (supra). À noter ici que les deux éléments composant ce poinçon, installés en sous-ouure sans démontage de la ferme, sont issus de la mème poutre (fig. 15).

En dépit du perit nombre d'éléments signalant la consolidation de la structure 50 à 70 ans sculement après sa mise en place, cette phase n'en est pas moins significarive des techniques de construction en bois du XI" siècle.

\section{LES MARQUES D'ASSEMBLAGES}

Une série continue de marques d'assemblages en chiffres romains, inscrites a la mine de plomb "', a été repérćc sur les assemblages d'origine des demi-fermes de la croupe. Elle progresse de I à XIII en partant au nord a la demi-ferme 13 jusqua a la demi-ferme 1 au sud. Ces marques sont inscrites sur la face interne des bois, sur les cherrons nord au-dessus des cavités destinées aux têtes des contrefiches et sur les cheurons sud au droir des mi-bois réservés aux jambes de force et aux demi faux entraits. Ces derniers reproduisent la marque au droic du mi-hois du chevron. en sous-face. Notons quaucune marque n’a été relevée sur les chevrons sud au

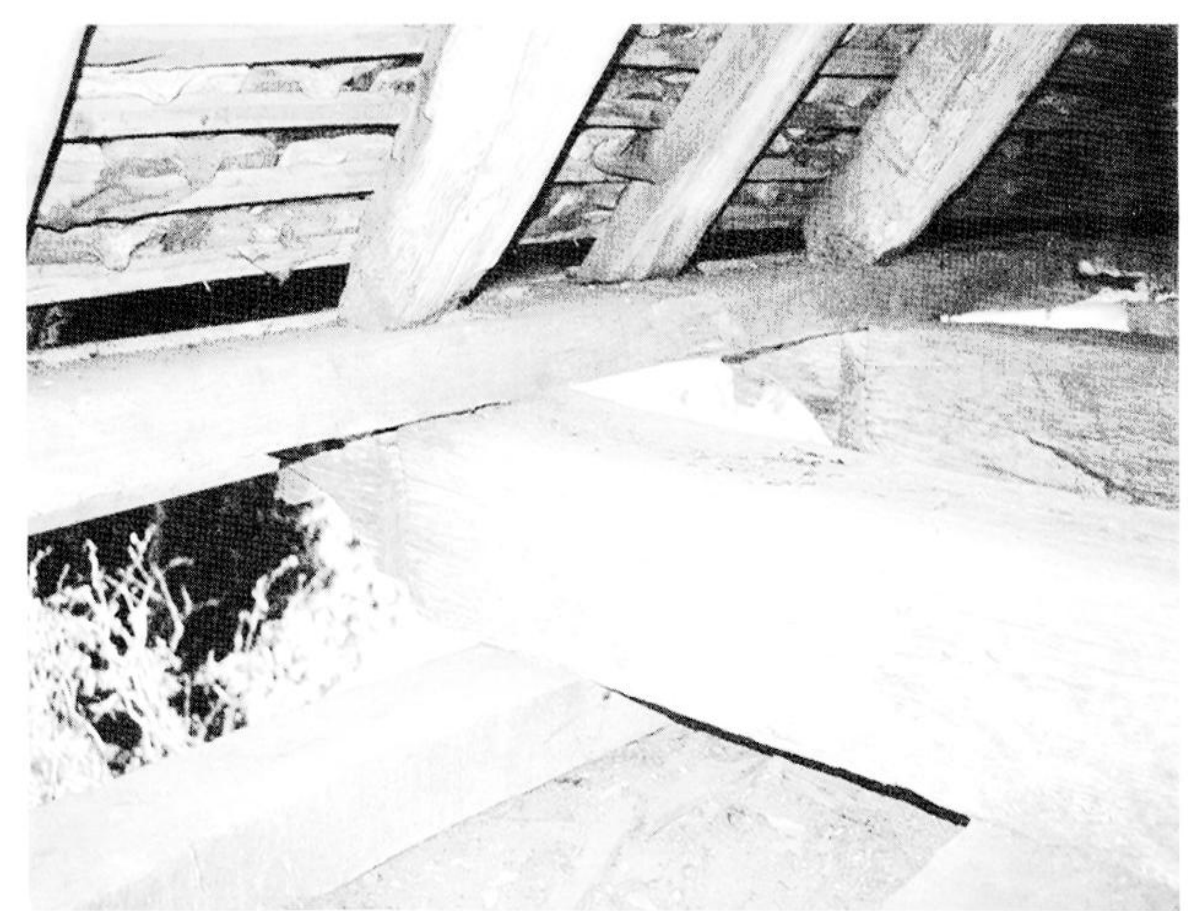

lïg. 17 - Vúc intéricure des sculpeures des abouts dentraits. 
niveau des mi-bois destinés aux potealux, preure que les charpenticers ont maryuce les assemblages apress šetre rendu compre du défaut de conception de ces demifermes. (e marquage sest done effectué apress la "mise-dedans ", opérarion qui consiste à assembler au sol les ćléments d'une ferme pour corriger les éventuels défauts de taille. (ees marques, présentes uniquement sur les assembiages d'origine, rémoignene d'une mise en place commune des demi-fermes nord et sud. bien que ces dernieres fussent taillées arant les demi-fermes nord.

Ce tepe de marpuage en chiffres romains est particulierement précoce au regarded des maryuages de la période romane caractérisés par des signes non ordinaux qui ne permettent pas de positionner une ferme au sein d'une numérotation continue. le systeme des chiffres romains va se répandre surtout a partir de la fin du Xil siecle aree le développemene des charpentes à structure traméc '”, qui réclament un positionnement spécifique pour chaque ferme er donc l'emploi d'un marquage ordinal.

Sur les fermes de la travée droite, ancune marque au graphite n'a éré relevée. Seul le cherron sud de la ferme 6 présente une marque en forme de signe particulier tracé à la pointe d'un ciscau a bois, sur sa face cst au-dessous du mi-bois du faux-eritrait. Bien qu'elle soit isolée, elle témoigne de l'usage d'un marquage non ordinal des fermes, typique de ces charpentes dont les fermes sont toutes identiques et interchangeables 2 .

\section{QUEL TYPE DE BOIS EMPLOYÉ POUR LA CHARPENTE ?}

À partir du profil de croissance caractérissique des échantillons dendrochronologiques, il est possible de déterminer le type de formation forestière exploité au XI' siècle autour de Neufmarché. En effer, la forte producrion radiale des jeunes tiges en phase juvénile "', suivie d'une brusque

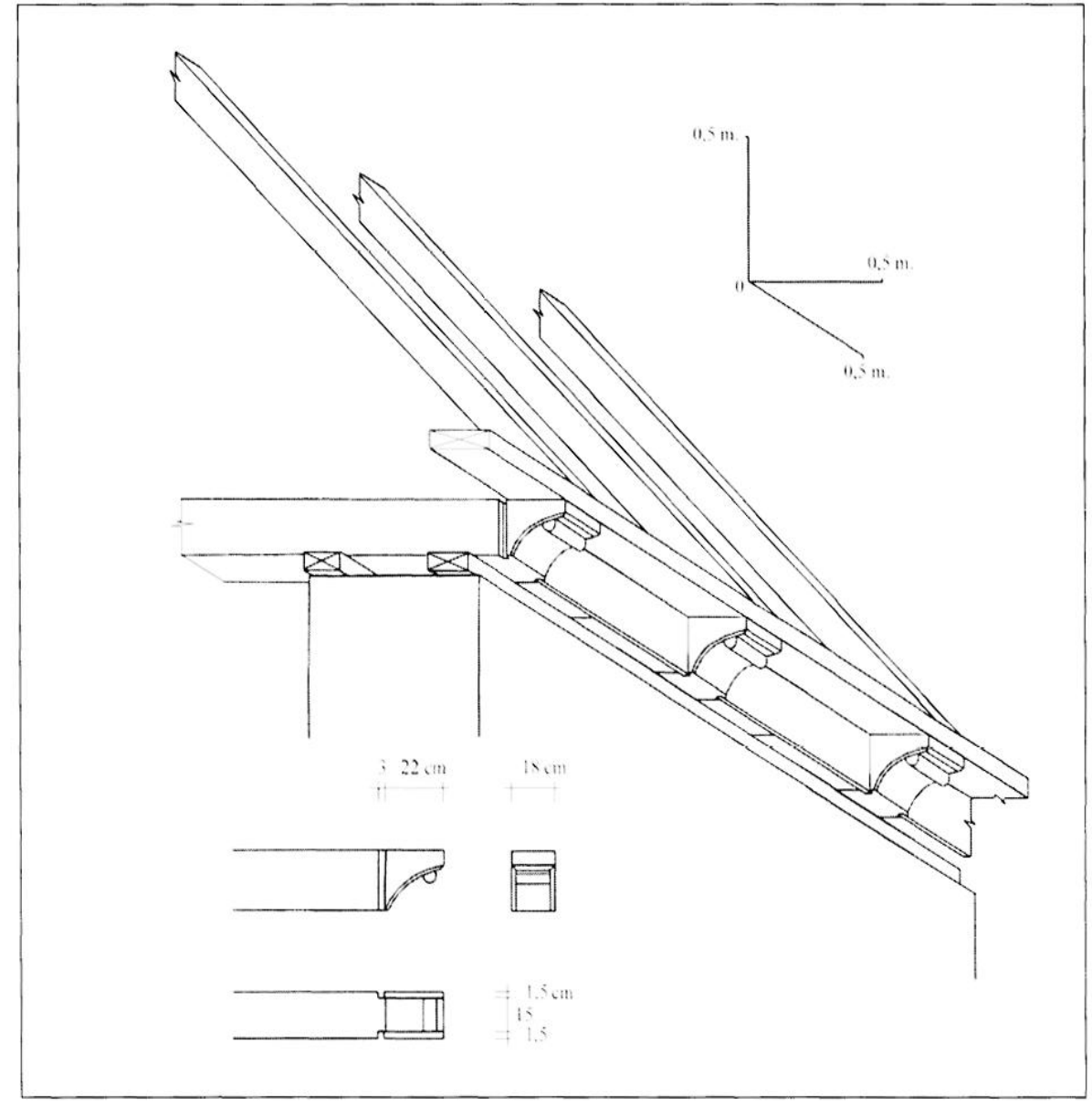

Fig. 18 - Sculptures des abouts dentraies (dessin 1: 1:paud).

chute de croissance (inférieure au seuil du mm) ne laisse aucun doute guant à un mode de régénciarion forestière par rejer de souche. Cette observation est relavée par le bon synchronisme de plusieurs séquences, non pas seulement par rapport a leurs derniers cernes, mais aussi par rapport à leurs premiers cernes (fig. 14). Ceci suggère une phase de régénérarion forestière rapide et homogène chronologiquement, comme dans le cas des taillis-sursouche. Cette phase de régénération des souches est elle-mème probablement consécutive à une phase d'abattage antérieure, vers 980, perceptible ici seulement en négatit. Cette perspecrive indique une occupation et une gestion précoce, du milieu forestier, en relation possible avec la collégiale ou avec le château de Neufmarché. Mais, il s'agit là des seuls éléments tangibles pour aborder cette occupation puisque aucun vestige de bois en élévation remontant à la fin du $X$ "siècle n’a éré découvert à ce jour.
Issu de ceete meme phase de régénération, larbre qui seral utilisé près dun siècle plus tard, a la transition des XI et Xils sieckes pour façonner le poinçon de la ferme 8 (NM15 et 16), est épargné lors de la phase dabattage de 1055-1070. Cet arbre de réserve, provenant probablement des mèmes souches que les autres bois utilisés à Neufnarché, illustre un mode de gestion forestière simple, qui caractérise véritablement l'ensemble de la période médiévale.

Parmi tous les échantillons synchronisés er datés, plusieurs d'entre eux présentent d'excellents niveaux de concordance, à tel point quil est difficile d'individualiser les courbes de croissance des demi-entraits 8 Est et 13 Nord (NM09 et 10) ou du demi-entrait 6 Est et du chevron du demientrait 9 (NM07 et 18)(fig. 15). Il parait alors évident que ces échantillons provenant de 4 pièces différentes sont issus en réalité de 2 arbres seulement. Cetre parcimonie dans l'urilisation des ressources 


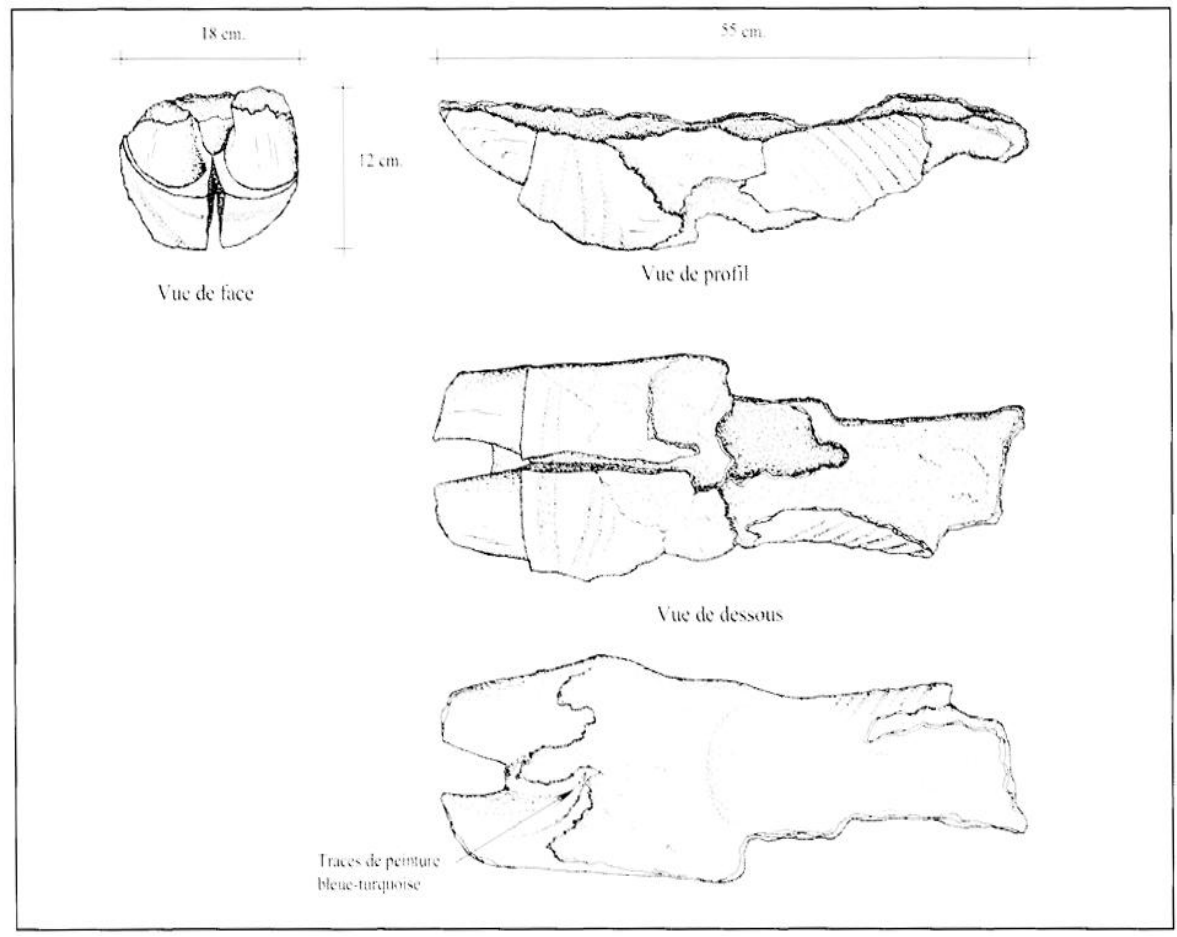

Fig. 19) - Sculprure dabout dentrait (dessin F. F.paud).

ligneuses fut réitérée au moment de la restauration de cette charpente, au XI'III siècle, lorsque la plupart des pièces d'origine furent réemployées.

\section{L'ORNEMENTATION EXTÉRIEURE}

Tous les demi-entraits de la croupe se prolongent au-delà des sablières pour déborder de $25-30 \mathrm{~cm}$ à l'extérieur du mur de l'abside, sous le débord de la toirure (fig. 16 et 17). Ces extrémités, visibles de l'extérieur, présentent toutes en sous-face une sculpture en forme de gorge incurvée, chanfreinée aux arêtes et pourvue d'un petit tonnelet horizontal, a l'exception des demientraits 10,11 et 12 mis en place en 1708 .

Au droit du parement extcrne du mur, les flancs de ces demi-entraits sont creusés d'une rainure verticale, profonde et large de 3 centimètres, destinće à recevoir un cache-moinealu. Il sagissait de planchettes disposées en entrevous des demi-entraits, en applique des sablières curvilignes, pour cloisonner l'espace compris entre la saillie des demi-fermes et assurer ainsi lisolement du comble (fig. 18).
Comme les extrémités des demi-entraits de la croupe, les entraits des fermes posées sur la travée droire du chœur faisaient également saillie à l'extérieur des murs, sous le débord de la toiture. Le cours des pièces de réception des pieds de cherrons se poursuivait sur l'about des entraits des fermes en sintercalant avec les cache-moineaux glissés en entrevous qui garantissaient le cloisonnement de ces saillies.

Sur la travée droite, tous les abouts des entraits ont été sciés lors des restaurations du X'I'III siècle, mais l'une de ces extrémités a été retrouvcée dans le comble de la chapelle latérale sud. Ce morceau de bois, extrèmement dérérioré, présente une sculprure en forme de tête zoomorphe pourvie d'un cou plissé, de deux yeux cernés prolongés d'un rostre à deux becs (fig. 19). La face de cette tête devait se présenter en face inférieure de l'entrait pour être visible d'en bas, comme pour les sculptures de la croupe. Cette tète conserve encore des traces résiduelles d'un enduit blanc sur lequel on remarque quelques fragments d'une couche de peinture bleueturquoise. Aucune trace d'enduit peint n'a cependant été remarqucé sur les sculprures encore en place de la croupe, exposées depuis le Xr siècle aux intempéries. l'état de conservation de ces traces de peinture et la difficulté de procéder à une retouche picturale sur ces éléments de bois en haut des murs gouttereaux semblent confirmer leur authenticité.

Espacés de $54 \mathrm{~cm}$ sur la travée droite du chour et de $61 \mathrm{~cm}$ en movenne sur l'abside, ces abouts d'entraits sculptés et peints, faisaient office de modillons pour le haut du mur. Ces charpentes de comble, dont les entraits étaient apparents à l'intérieur sous la forme d'un plafond, rentraient dans la composition ornementale de l'édifice et leur débord renvovait à l'extéricur de l'église, sous forme décorative, la trame de ces plafonds apparents.

De tels modillons en bois ne sont pas exceptionnels pour la période romane. Au sommet des murs gouttereaux de l'eglise de la Basse-CEuvre de Beauvais, du $x$ siècle, les encraits rapprochés de l'ancien plafond apparent débordent à l'extérieur. avec des sculptures encore visibles sur certains abouts ".". Sur la nef de l'église SaintGermain à Allouis dans le Cher, les entraits de la charpente datée par dendrochronologie de 1125 débordent également à l'extérieur des murs ". À Chabris dans l'Indre, sur le croisillon nord de l'église Saint-Christophe, un entrait réemployé de la charpente du xl siècle conserve à l'une de ses extrémités une rainure de cachemoineau qui témoigne de son surplomb a l'origine sur les murs gouttereaux $: 2$. Ce sont parfois les blochers qui débordent de $30 \mathrm{~cm}$ environ au-delà des murs comme a Monthou-sur-Cher dans le Loir-et-Cher, sur l'abside de l'église Saint-lié darée par dendrochronologie de 1173, et à CourCheverny dans la mème département. sur l'église Saint Aignan darcée de 1159 par dendrochronologic ${ }^{\sharp}$. Dans ces derniers exemples, les abouts débordants ne présentent aucunc sculpture, mais les bois sone bien apparents en surplomb a l'extérieur comme des modillons en pierre et ils comportent tous des vestiges de cache-moineaux glissés dans des rainures sur leurs flancs pour le cloisonnement du comble. 
Il subsiste encore en Suide, dans les églises du XII siècle de Kumlab! a Visingsö et de Väversundia, des charpentes a chevrons-formant-fermes romanes dont les entraits débordent en saillic à l'extéricur des murs ". De mème en Belgique, à Liège, dapres les propositions de restiturion des charpentes primicives de la nef de l'église Saint-I)enis (1015-1020d) et de l'église Saint-Barthélémy (vers 119)d) ".

Il est permis de se demander si ces modillons en bois imiene des modillors en pierre ou si ces derniers ne sont pas all contraire la forme " pécrifice o de ces sculptures en bois, plus fríquentes quion ne ke supposerait sur les édifices antérieurs au vil siecke. ()n peut effectivement remaryuer que les modillons en pierre reproduisent l'ccartement de 50 a $60 \mathrm{~cm}$ des fermes rapprochées des charpentes romanes qui, dans la grande majorité des cas, étaient pourrues dun entrait a leur base jusquau milieu du XII sièce dans la moiric nord de la France. Dans lhistoire de larchitecture, comme dans l'histoire de larr, il est fréquent de voir se perpétuer des formes au cours des siècles a travers l'usage de différents matériaux ou de nouveaux supports. Les modillons de pierre pourraient peut-itre rípondre de ce processus de " pétrification " des formes de l'architecture de bois. De nombreux frontons dédifices antiques présentent ainsi sous les rampants et sur l'entablement, des modillons en pierre qui reproduisent la position ex la forme des extrémités des pannes de la coirure er des solives d'un plafond en bois. Le pignon en pierre de lauvent des basiliques paléochrétiennes de Brïd et de Baruta en Syrrie du Nord "représentent même une ferme triangulée dont on distingue parfaitement l'entrait, les deux arbalćtriers, le poinçon et deux contrefiches obliques.

D) autre part, il est probable que le débord de la toiture de ces édifices était destiné à protéger du ruissellement pluvial un décor appliqué sur la partie haute du mur. La charpente de l'abside de l'église de Monthoul-sur-Cher, datée de 1173, protège ainsi un décor appareillé géométrique très soigné, limité au tiers supérieur du mur, au-dessus des baies et du contrefort axial (fig. 20). Sur l'abside du choxur romann de l'́glise de lye dans l'Indre, le débord de la charpente permet aussi de protéger un bandeau de peintures médiévales situce en haut du mur, sous le débord de la toiture ".

Il est donc probable quà Neufmarchéen-lyons le débord des entraits servait sigalement à la protection d'un décor peint. aujourd hui disparu, qui expliquerait alors la présence du bandeau de maçonnerie en moyen apparcil tries régulier, limité au tiers supéricur du mur, all-dessus du calus des contreforts et des baies (fig. 2). less modillons de bois sculprés et peints participaient aussi vraiscmblablement à cette ornementation extéricure.

\section{CONCLUSION}

Si l'excmple de Neufmarché-en-lyons nautorise aucune généralisation, il permet néanmoins d'échafauder de nourelles hrpothises sur le décor extérieur des églises romanes. l'extreme rareté des charpentes du Xi siécle encore en place ne peur que stimuler notre attention a leur égard mais doit également nous inciter a la plus grande prudence quant à keur interprétation et a leur analyses.

La construction du cheve de l'église de Neufmarche et la mise en place de cette charpente lors de la transformation de la

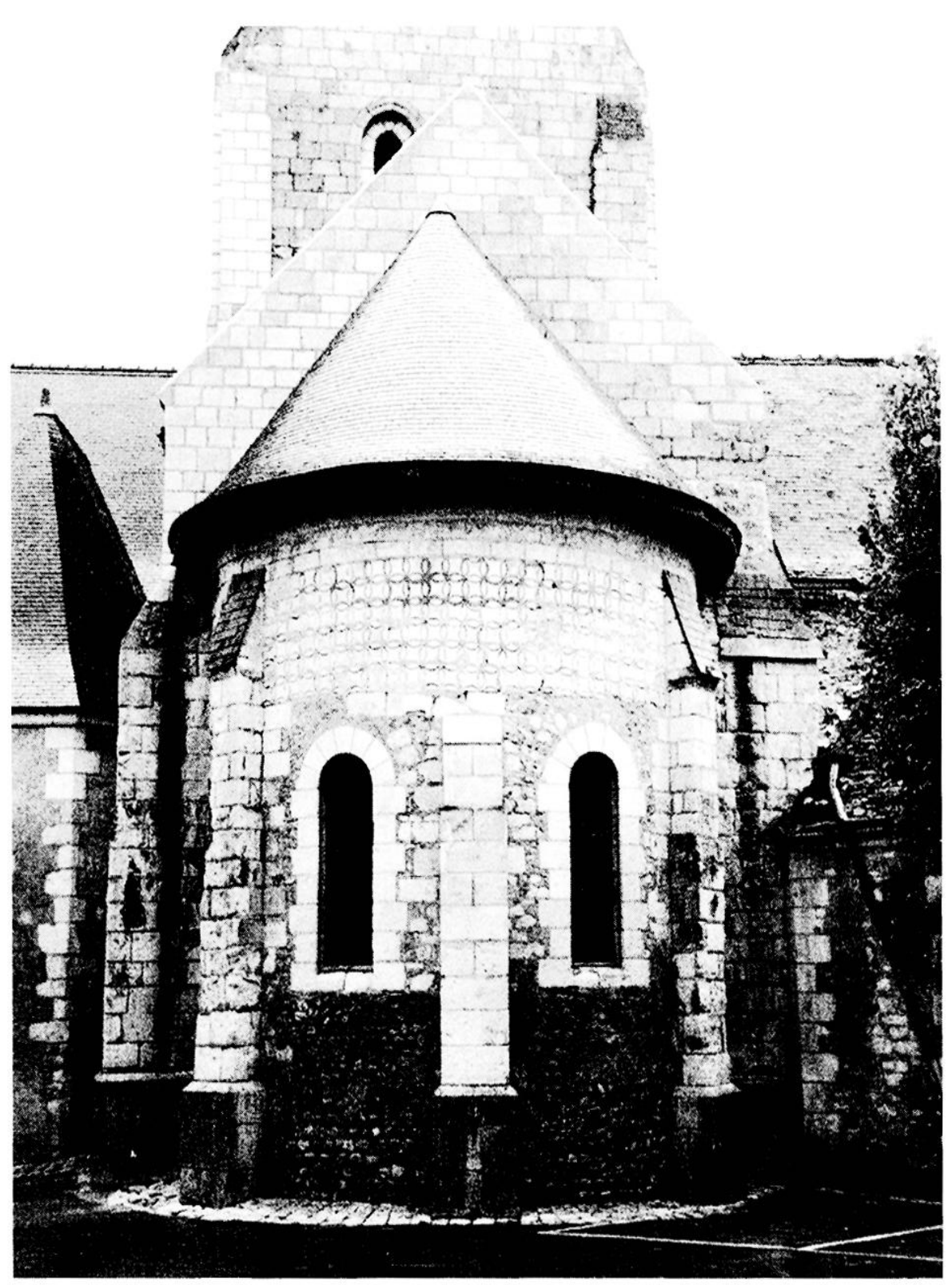

Fig. 20 - Abside de l'église Saint-lié de Monthou-sur-Cher (I.oir-et-Cher). 
collégiale en pricuré par Hugues de Grandmesnil. attestece par les textes vers 1065 er par la dendrochronologie entre 1055 ex 1070, constituent un nouvel étalon de référence pour l'étude de l'architecrure romane normande et illustrent avec éridence lintérêt de mettre en corrélation léeude des charpentes et celle des structures maçonnées sous-jacentes. De méme, il importe de siattarder particulièrement. dans létude du baiti médiéval, sur les rares témoins de la réflexion cechnique des charpenticrs qui transparaissent au travers des erreurs de conception, des défauts de raille er de certaines opérations de préparatoon des bois comme le marquage des assemblages. Ces indices fugaces permettent en effet de percevoir le mode de réflexion rechnique des charpentiers du XI siecle er de suivre le processus mental de ces hommes lors de la conception et de Pélaboration de la charpente. Au-delà de lérude des rechniques et des structures, cetre démarche tente arant tout de comprendre leur ćrolution à travers la perception de cette pensée technique.

\section{NOTES}

Tous les diches sone de Frideric lipaud.

1. Cous renons a remercicr la Conscration Regionale des . Monuments Historiques de HauteVormandic en la personne de l.. Dumarche. (harge dPfudes Documentaires pour nous aroir communique la dicourerte de certe charpente et l. Iescroart, Conservateur Regional des Monuments Historiçuts, pour be finuniemene des datacions dendrochronologicyues.

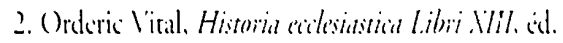
(iuizot. (akn. 1826, t.2. p. 2).

3. 16 rid. p. 108 .

t. Hiel. p. 108 .

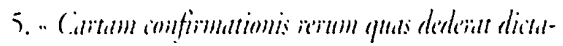

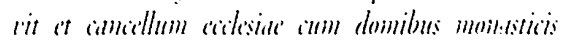
ienormit. ", ibidem. r.t. p. +1t.

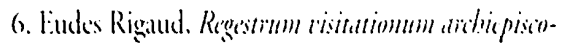

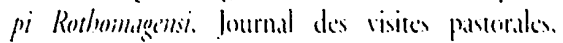

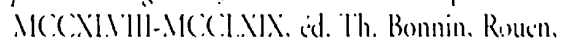
1852. p. +1.3

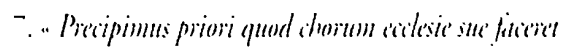

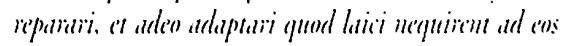

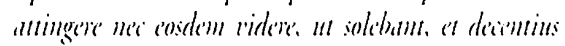

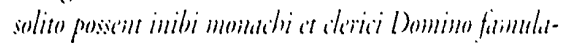
ni. ibielem. p. 620.

8. Arch. I)cip. Scinc-Maricime. (i. St? ().

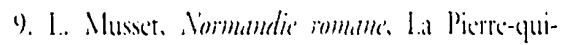
Yire. t.2. 1985. p. 29.3.

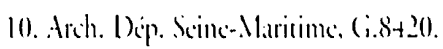

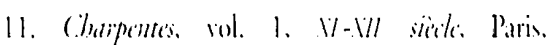

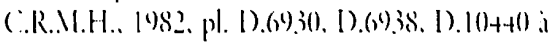
D.11)+4.3. Rapport danalues de dendrochromologie sur Saint-(icrmain-den-Pres: P. Praiture a

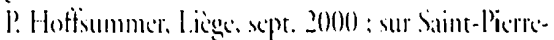

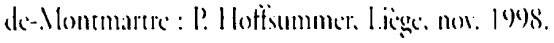

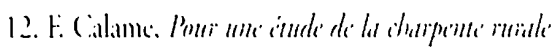

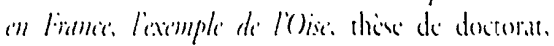

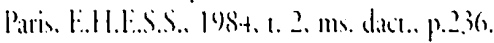

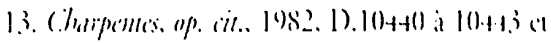

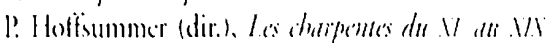

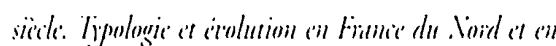

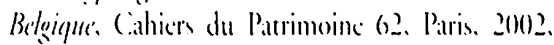
p. is

14. lépuarrisage de cos sablieres curvilignes et leur parfait ctat de comscration trahissent une mise en place recente. Il en va de mime pour le cours des sablieres formant plate-forme dome les bois ont etri cyuarris a la scie de longe ce yui ne preseneme ancolne trace dusure ou dalremation. Il apparair cepen-

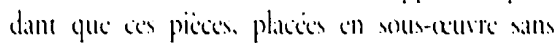
dimonatage des superstructures, epproduisent lancienne disposition des sablieres et des pieds de cherrons.

15. In remploi delemens de firmes principales at complitemente ciclu en maison des sculpeures diabour déneraie yui sone dorigine ce partaitemene adapreses i la courtxe de labside.

16. Cet assemblages situé en pied du poinçon. est fixé par ciny cherilles a consolide par trosis calles.

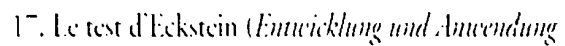

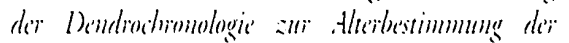

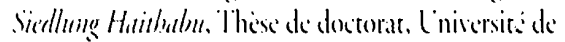
Hambourg. 196(9)). appedi plus friquemment esest " II" . comnritue un des calculs les plus urilisis en linrope accidentak. (e test. issu du calcul de parallélisme ricone comper de la longueur des coubes comparies. tinsi. ples les siguemes controlices scront longues plus la senchronisation scra sure. l'our las montages successifs de siquencise nous coartons les resultats infericurs a 3 . tin theoric. $k$

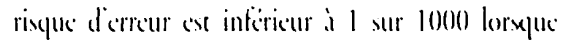

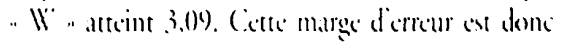
acieptable, surtout lorspue la contemporancite des

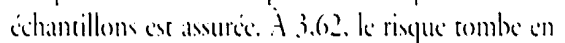
dewous de 1 sur 10 0000. P'ar concipuent. a partir de 4. kes resultars deriemnent ress fialles.

le cos . II" a pricente le grand axantage de rester indipendant de lamplitude des variation interannucllos a permet de comparer des courles de moyenne ere diamplitude ries diverses.

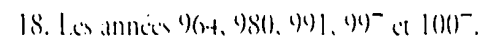

19. II finut noter que ce revultat ateribue unce anncic calendaire and lit corme de la monems

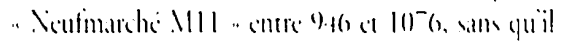

corresponde à une fourchere de datacion. relle cue le (: peut en fournir par exemple.

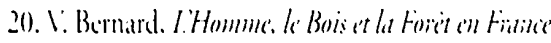

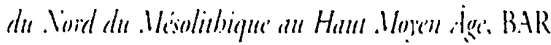
Internarional scrics. Hedges cid. () sterd, lives.

21. (i.-. L. Lmbert. I: Bernard at alii. "Fench regional o:k chronologies spanning more than 1000

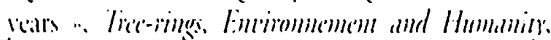

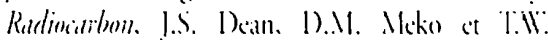
Siwcernam (dir.). Acres du congres innernationale de

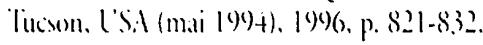

22. F. Hollstcin. " lahrringehronologische 1)aticruns won lïchenhölyern ohne Wakklkinte".

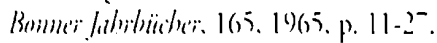

23. 1 ou 2 ans au grand maximum. Mais dans la

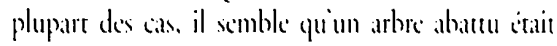
intigriansiror dans la conscruction.

2.t. ()ans "yino des cals.

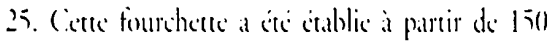
abbiers complets provenant de bois midiciaux de la Scinc-Maritimes de l'Fure du Calvados er de la Manche.

26. Appelic auss graphite ou plombaginc.

$2-$ ( Charpentes done la structure se divise en enalises regulieres maryuces par des fermes principales et sccondaires adaptes a la trame du baitiment.

28. lixemple de marepuage non ordinal : charpence remplovede de la net de la carhedrate de Rouen de la fin du vir sick charpene de la dhapelle de lancien Palais Fipiscopal de laton datcic par dendrochronologice enere $11^{-8}$ er 1188 : charpente du briss sud du tranxpe de la carthedralc de lisicux du premier

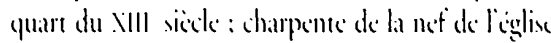

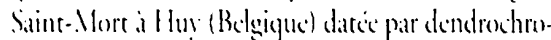
nologic enere 1230 a t 12.35: charpente du chesur de

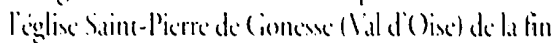

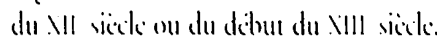

29. Dams les 10 a 15 premieres ammes de leur developpementer.

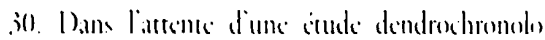

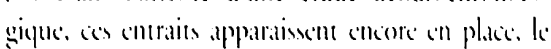




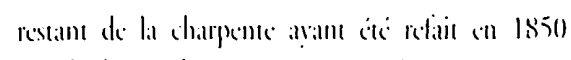

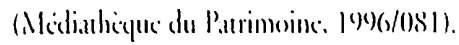

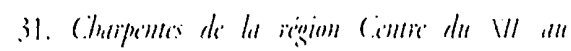

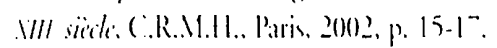

32. Ibirl. p. 10-1.t.

3.) Ilide p. 18.5.

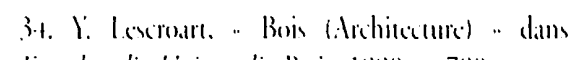

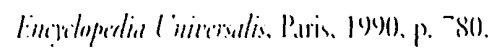

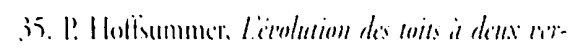

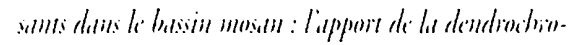

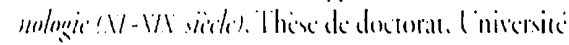
d. liegr. 198\%, m. dact. vol. 1. p. 58 .

36. I.P. Sodini, " Los premiers manilestatom de la

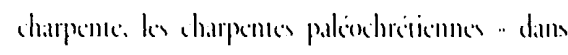

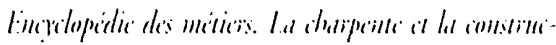

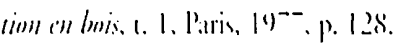

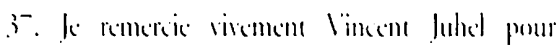

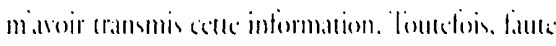

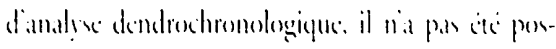
sible de virifier lanciennek de lis charpente. 